\title{
ON HOLOMORPHIC POLYDIFFERENTIALS IN POSITIVE CHARACTERISTIC
}

\author{
SOTIRIS KARANIKOLOPOULOS
}

\begin{abstract}
In this paper we study the space $\Omega(m)$, of holomorphic $m$-(poly)differentials of a function field of a curve defined over an algebraically closed field of characteristic $p>0$ when $G$ is cyclic or elementary abelian group of order $p^{n}$; we give bases for each case when the base field is rational, introduce the Boseck invariants and give an elementary approach to the $G$ module structure of $\Omega(m)$ in terms of Boseck invariants. The last computation is achieved without any restriction on the base field in the cyclic case, while in the elementary abelian case it is assumed that the base field is rational. An application to the computation of the tangent space of the deformation functor of curves with automorphisms is given.
\end{abstract}

\section{INTRODUCTION}

Let $F$ be an algebraic function field with field of constants $K$, where $K$ is an algebraic closed field of characteristic $p$. Let $F / E$ be a Galois extension with abelian Galois group $G$ of order $p^{n}$. We will denote by $\Omega_{F}(m)$ the space of holomorphic $m$-(poly)differentials of $F$. We know that $\Omega_{F}:=\Omega_{F}(1)$ is a $g_{F}$-dimensional $K$-space, while the $\Omega_{F}(m)$, is a $(2 m-1)\left(g_{F}-1\right)$-dimensional $K$-space, when $g_{F} \geq 2$. The Galois module structure of the space of holomorphic 1-differentials has been determined explicitly in some cases. The cyclic group case was studied by Hurwitz [9] if the characteristic of $K$ is zero. When $F / E$ is unramified and $G$ has a prime to $p$ order, or is a cyclic group, Tamagawa [20] proved that is the direct sum of one identity representation of degree one and $g_{E}-1$ regular representations. Valentini [22] generalized this result for unramified extensions having $p$-groups as their Galois groups, while Salvador and Bautista [14] determined completely the semisimple part of holomorphic differentials when $G$ is a $p$-group. If $G$ is cyclic then Valentini and Madan [23], determine completely the whole structure of $\Omega_{F}$ in terms of indecomposable $K[G]$-modules. The same is done when $G$ is an elementary abelian, by Calderón, Salvador and Madan [17]. Also N. Borne [2] developed a theory, using advanced techniques from modular representation theory and $K$-theory, and he is able to compute the $K[G]$-module structure for holomorphic $m$-differentials $\Omega_{F}(m)$, when $G$ is a cyclic group of order $p^{n}$. In general the $K[G]$-module structure of $\Omega_{F}$, in positive characteristic is unknown. The difficulties that arise in positive characteristic, in contrast to the same problem in characteristic zero, are first all the difficulties of modular representation theory, in contrast to ordinary representation theory, and second the appearance of wild ramification in extensions $F / E$.

In this paper we will mainly focus on the $m>1$ case and on the two "extreme" cases of abelian groups of order $p^{n}$ namely cyclic groups and elementary abelian groups. We first compute a basis for holomorphic differentials and then we define the quantities $\nu_{i k}(m)$ to be

$$
\left\lfloor\frac{m \delta_{i}+\{\text { evaluation of the kth E- basis element of } \mathrm{F} \text { by a normalized valuation of } \mathrm{F}\}}{p^{e_{i}}}\right\rfloor,
$$

Date: October 30, 2018.

keywords: Automorphisms, Curves, Differentials, Galois module structure, AMS subject classification $14 \mathrm{H} 37,11 \mathrm{G} 20$. 
where $\delta_{i}, e_{i}, i$ are related with the ramification of the extension, see Conjecture 26 below and Remark 16, the basis element is evaluated by a (normalized) valuation determined by a place of $F$ above a ramified place of $E$ and $\lfloor\cdot\rfloor$ denotes the integer part. We introduce the Boseck invariants that are quantities of the form $\Gamma_{k}(m):=\sum_{i} \nu_{i k}(m)$, where the index $i$ runs over all ramified places. These quantities were used by Boseck [3] for constructing bases for 1-holomorphic differentials and the conditions for holomorphicity are expressed in terms of them. In order to find Bosek's invariants for the function fields $F / E$ that we study, we take the rational extensions $F / K(x)$ and find $K$-bases for the corresponding $\Omega_{F}(m)$ 's for $m \geq 1$ (when $m=1$ these bases often called Boseck bases). The choice of the rational function field is clear each time from the defining equations of our curves (Eq. (6), (22), (23). It turns out that in the cases we study, the Boseck invariants determine the Galois module structure of the space of $m$-holomorphic differentials. The formula that gives this $K[G]$ module structure in terms of the Boseck invariants remains the same in both the elementary abelian and the cyclic case. In addition in the cyclic case this formula is independent from $r$, with $r$ measuring how high in the tower of intermediate fields is placed an unramified subextension $E_{r} / E$. This is not true when $m=1$ (see Remark 8). The elements $\Gamma_{k}(m)$ carry a lot of information, for instance the degree of the different of the extension each time, can be described totally by them (see Remarks 7, 14, 18, namely

$$
\frac{2}{2 m-1} \sum_{k} \Gamma_{k}(m)=\operatorname{deg} \operatorname{Diff}(F / E) .
$$

Using Boseck invariants, we describe every $K[G]$-module structure of holomorphic $m$ (poly)differentials $\Omega_{F}(m)$ for $m \geqslant 1$, when: $G$ is cyclic (for $m=1$ case this is [23], Theorem 2]), or elementary abelian of degree $p^{n}$ (for $m=1$ case this is [17, Theorem 1]). Finally we show how the Boseck basis in the $m=1$ case when $F / E$ is tame, i.e, of degree $n$, with $n$ being prime to $p$, will give another proof of the classical Hurwitz Theorem [9, $\mathrm{p}$. 439, formula 2].

Our approach, which is quite elementary, follows closely the ideas of Valentini- Madan, Calderón,Villa-Salvador and Madden [16]. Madden, used the same analysis and constructed a $K$-basis of $\Omega_{F}(1)$, in order to compute the rank of the Hasse-Witt matrix. We should mention here that all the above authors had used Boseck invariants in their papers [23], [17], [16].

The organization of the paper is as follows: In first section we focus on the cyclic case and give the Galois module structure of $\Omega_{F}(m)$ in Theorem 6 in subsection 2.1 As our analysis is going deeper, the ideas of Boseck [3] are rising up. We follow him, in subsection 2.2, in order to give a basis for $m$-holomorphic (poly)differentials of $F$, when $E$ is rational. Subsection 2.3 is devoted to a proof of the classical result of Hurwitz mentioned above. In section 3 we consider the elementary abelian case. At first we give an analogous $K$ basis for $\Omega_{F}(m)$. These bases can lead to the computation of $m$-Weierstrass points (see Remark 20. . Our proof of theorem 21 in subsection 3.2 is based on the work of Calderón, Salvador and Madan [17].

In section 4 we state a conjecture concerning the Galois module structure of $m$ - differentials when $G$ is a general abelian extension of order $p^{n}$.

Finally, in section 5 we give an application to the computation of the tangent space of the local deformation functor in the sense of J.Bertin and A. Mézard [1]. The results are given in terms of the Boseck invariants, and coincide with computations done previously by other authors [1],[13],[11],[12] using completely different methods. This allows us to verify our complicated computations concerning the Galois module structure. Also proving the conjectures stated in section 4 will give a method in order to compute the above mentioned dimension in the case of abelian groups, a problem that is still open.

An other application we have in mind and we would like to explore in a following article is the computation of higher order Weierstrass points and the study of the fields 
generated by the coefficients of them, a problem that is similar to the classical study of fields generated by torsion points of the Jacobian, [18], [21].

In order to avoid trivial cases we will always assume that $g_{F} \geq 2$, where $g_{F}$ is the genus of $F$. We use the symbol $\mathbb{P}$ to denote the set of places (sometimes referred just as "primes") of the field in question.

\section{The Cyclic CASE}

Since the characteristic $p$ divides the order of the group $G$, the representation of $G$ on $\Omega_{F}(m)$ is not necessary completely reducible, but it is the direct sum of indecomposable $K[G]$-modules. Let $\sigma$ be a generator for $G$. The unique indecomposable $K[G]$-module of degree $k$ is isomorphic to $K[G] /\left\langle(\sigma-1)^{k}\right\rangle[24$, p.156, Ex. 1.1 ]. For $k=1$ we obtain the identity representation and for $k=p^{n}$ the regular representation.

Let

$$
\Omega_{F}(m):=\bigoplus_{\lambda=1}^{t} W_{\lambda},
$$

be a decomposition into a direct sum of indecomposable $K[G]$-modules and let $d_{k}$ be the number of $W_{\lambda}$ 's that are isomorphic to $K[G] /\left\langle(\sigma-1)^{k}\right\rangle$. We will compute the $d_{k}$ 's. First we define

$$
\Omega_{F}^{i}(m)=\left\{\omega \in \Omega_{F}(m):(\sigma-1)^{i} \omega=0\right\}, \text { for } i=0,1, \ldots p^{n} .
$$

These $K$-subspaces form an increasing sequence with $\Omega_{F}^{0}(m)=0$ and $\Omega_{F}^{p^{n}}(m)=\Omega_{F}(m)$, while

$$
\operatorname{dim}_{K} \Omega_{F}^{i}(m)=\sum_{\lambda=1}^{t} \operatorname{dim}_{K}\left(W_{\lambda} \cap \Omega_{F}^{i}(m)\right) .
$$

Lemma 1. We have $\operatorname{dim}_{K} K[G] /\left\langle(\sigma-1)^{k}\right\rangle=k$.

Proof. Consider the map $(\sigma-1)^{k}: K[G] \rightarrow K[G]$. Observe that $\operatorname{dim}_{K} \operatorname{ker}(\sigma-1)^{k}=$ $k$ therefore $\operatorname{dim}_{K} \operatorname{im}(\sigma-1)^{k}=p^{n}-k$ and the quotient $\operatorname{dim}_{K} K[G] /\left\langle(\sigma-1)^{k}\right\rangle=$ $\operatorname{dim}_{K} K[G] /\left\langle\operatorname{im}(\sigma-1)^{k}\right\rangle$ has dimension $p^{n}-\left(p^{n}-k\right)=k$.

\section{Lemma 2.}

$$
\operatorname{dim}_{K}\left\{\alpha \in K[G] /\left\langle(\sigma-1)^{k}\right\rangle:(\sigma-1)^{i} \alpha=0\right\}= \begin{cases}i, & \text { if } i \leq k, \\ k, & \text { if } i \supsetneqq k\end{cases}
$$

Proof. Indeed, we would like to compute the kernel of the multiplication with $(\sigma-1)^{i}$,

$$
(\sigma-1)^{i}: K[G] /\left\langle(\sigma-1)^{k}\right\rangle \rightarrow K[G] /\left\langle(\sigma-1)^{k}\right\rangle .
$$

We distinguish the following two cases:

(1) If $i \leq k$ then $\operatorname{ker}(\sigma-1)^{i}=(\sigma-1)^{k-i} K[G] /\left\langle(\sigma-1)^{k}\right\rangle$ and $\operatorname{dim}_{K}(\sigma-$ $1)^{k-i} K[G] /\left\langle(\sigma-1)^{k}\right\rangle=p^{n}-(k-i)-\left(p^{n}-k\right)=i$.

(2) If $i \geq k$ then $\operatorname{ker}(\sigma-1)^{i}=K[G] /\left\langle(\sigma-1)^{k}\right\rangle$ and $\operatorname{dim}_{K} K[G] /\left\langle(\sigma-1)^{k}\right\rangle=k$ according to Lemma 1

Using the decomposition of $\Omega_{F}(m)$ given in Eq. (2) and Lemma 2 we obtain $\operatorname{dim}_{K} \Omega_{F}^{i}(m)=\sum_{k=1}^{i-1} k d_{k}+\sum_{k=i}^{p^{n}} i d_{k}$. So

$$
\operatorname{dim}_{K}\left(\Omega_{F}^{i+1}(m) / \Omega_{F}^{i}(m)\right)=\sum_{k=i+1}^{p^{n}} d_{k}
$$


Therefore, for $k=1, \ldots, p^{n}-1$ we have:

$$
\begin{aligned}
d_{p^{n}} & =\operatorname{dim}_{K}\left(\Omega_{F}^{p^{n}}(m) / \Omega_{F}^{p^{n}-1}(m)\right), \\
d_{k} & =\operatorname{dim}_{K}\left(\Omega_{F}^{k}(m) / \Omega_{F}^{k-1}(m)\right)-\operatorname{dim}_{K}\left(\Omega_{F}^{k+1}(m) / \Omega_{F}^{k}(m)\right) .
\end{aligned}
$$

Following [23] we write down a convenient $E$-basis for $F$ and we find the $G$-action on the basis elements and state the main Theorem.

Since $F / E$ is cyclic of degree $p^{n}$, there is a tower of intermediate fields

$$
E=E_{0} \subset E_{1} \subset E_{2} \subset \cdots \subset E_{n}=F,
$$

where each of the $E_{j} / E_{j-1}$ is an Artin-Schreier extension given by

$$
E_{j}=E_{j-1}\left(y_{j}\right), y_{j}^{p}-y_{j}=b_{j}, b_{j} \in E_{j-1}, 1 \leq j \leq n .
$$

The elements $b_{j}$ are called to be in standard form, for a place $P$ of $E_{j-1}$, if the valuation of the divisor of $b_{j}$ at $P$ is positive, zero or relatively prime to the characteristic $p$.

The first part of the following Theorem is due to Madden, and allows us to take $b_{j}$ 's in standard form [16, Theorem 2, p. 308], while the other part is due to R. Valentini and M. Madan [23, Lemma 1, p. 109] and allows us to select a convenient $E$-basis of $F$.

Theorem 3. The elements $y_{j}$ and $b_{j}$ can be selected so that:

(1) For any place $P$ of $E_{j-1}$ divisible by a ramified place in $F / E$ the valuation of $P$ of the divisor of $b_{j}$ is either zero or negative and relatively prime to $p$.

(2) $\sigma^{p^{j-1}}\left(y_{j}\right)=y_{j}+1$.

For $0 \leq k \leq p^{n}-1$ consider its p-adic expansion $k:=a_{1}^{(k)}+a_{2}^{(k)} p+\cdots+a_{n}^{(k)} p^{n-1}$ and denote by $w_{k}=y_{1}^{a_{1}^{(k)}} y_{2}^{a_{2}^{(k)}} \cdots y_{n}^{a_{n}^{(k)}}$. Then $F$ is an $E$ vector space with basis $\left\{w_{k}: 0 \leq\right.$ $\left.k \leq p^{n}-1\right\}$. The G-action on the $w_{k}$ 's is given by

$$
(\sigma-1)^{k} w_{k}=\prod_{\epsilon=1}^{n} a_{\epsilon}^{(k)} !
$$

This basis, has the following property

Lemma 4. Let $\bar{P}$ be a place of $E$ and let $P_{1}, P_{2}, \ldots, P_{r}$ be the places of $F$, above $\bar{P}$. Let $v_{i}$ the normalized valuation of $F$, determined by $P_{i}, i=1, \ldots, r$. Let also $b_{j}$ be in standard form for any place of $E_{j-1}$ below some $P_{i}$. If $z=\sum_{k=0}^{p^{n}-1} c_{k} w_{k}$, then $\min _{i} v_{i}(z)=$ $\min _{i, k} v_{i}\left(c_{k} w_{k}\right)$.

Proof. [23, Lemma 2, p.109] or [16, Lemma 3, p.310] .

2.1. Galois Module Structure of $\boldsymbol{\Omega}_{\mathbf{F}}(\mathbf{m})$. Let $\bar{P}_{i}, i=1, \ldots, s$ be the places of $E$ which ramify in $F$ and set $p^{e_{i}}:=e\left(P_{F} / \bar{P}_{i}\right), i=1, \ldots, s$ for the corresponding ramification indices, with $P_{F}$ a fixed place of $F$ above $\bar{P}_{i}$. We will denote by $\bar{v}_{i}$ the normalized valuation of $E$ determined by $\bar{P}_{i}$. Set $r=n-\max _{i}\left\{e_{i}\right\}$. We observe that $E_{r} / E$ is an unramified extension: if not then from the transitivity of the ramification indices we will have

$$
e\left(P_{F} / \bar{P}_{i}\right)=e\left(P_{F} / P_{E_{r}}\right) \cdot e\left(P_{E_{r}} / \bar{P}_{i}\right) \Rightarrow p^{e_{i}}=\left[F: E_{r}\right] \cdot p^{h} \Rightarrow p^{e_{i}}=p^{n-r} \cdot p^{h},
$$

where $h$ is a nonzero natural number. So $e_{i}=n-r+h \stackrel{r=n-\max _{i}\left\{e_{i}\right\}}{\Longrightarrow} e_{i}=\max _{i}\left\{e_{i}\right\}+h$ which contradicts the maximality of $e_{i}$.

Fix an $i$. Let $r_{i}=n-e_{i}$. Let also $P(i, j, \mu)$ be the places of $E_{j}$ which divide $\bar{P}_{i}$ and $v(i, j, \mu, z)$ be the normalized valuation of $E_{j}$ determined by $P(i, j, \mu)$, applied to an element $z \in E_{j}$. Each of the $E_{j} / E_{j-1}$ is normal and separable, so every one of the $P(i, j, \mu)$ 's will have the same exponent $d\left(P(i, n, \mu) / \bar{P}_{i}\right):=\delta_{i}$ in the different of $F / E$, Diff $(F / E)$. We can recover this different from the $\operatorname{Diff}\left(E_{j} / E_{j-1}\right)$, for all 
$j=1, \ldots, n$ using the transitivity property of the different (see Stichtenoth, [19, p.88, Corollary III.4.11.(a)])

$$
\operatorname{Diff}\left(E_{j} / E\right)=\operatorname{Con}_{E_{j} / E_{j-1}}\left(\operatorname{Diff}\left(E_{j-1} / E\right)\right)+\operatorname{Diff}\left(E_{j} / E_{j-1}\right) .
$$

Also, every automorphism of $F$ will act transitively on every place over $\bar{P}_{i}$, so the set $\operatorname{Diff}\left(E_{j} / E_{j-1}\right)$ is stable under $\sigma$, and the exponent of $P(i, j, \mu)$ in $\operatorname{Diff}\left(E_{j} / E_{j-1}\right)$ is independent of $\mu$. The following Lemma gives us the relation among $\delta_{i}$ and $b_{j}$.

Lemma 5. Let $\Phi(i, j)=-v\left(i, j-1, \mu, b_{j}\right)$. The different $\operatorname{Diff}(F / E)$ is given by

$$
\operatorname{Diff}(F / E)=\sum_{i=1}^{s} \delta_{i} \sum_{\mu} P(i, n, \mu),
$$

where

which equals to

$$
\delta_{i}=(p-1) \sum_{j=n-e_{i}+1}^{n}(\Phi(i, j)+1) p^{n-j}
$$

$$
\delta_{i}=(p-1) \sum_{j=1}^{n} \Phi(i, j) p^{n-j}+\left(p^{e_{i}}-1\right) .
$$

The valuations of the basis elements $w_{k}$ are given by

$$
v\left(i, n, \mu, w_{k}\right)=-\sum_{j=1}^{n} a_{j}^{(k)} \Phi(i, j) p^{n-j} .
$$

Proof. This comes from Proposition 2 of Madden [16], after replacing $j$, by $\nu+n-e_{i}$ and noticing that the different exponent does not depend on the choice of the base field (rational or not). However, we can prove it directly; fix an $i$ and apply the transitivity property of the different exponent $d\left(P(i, n, \mu) / \bar{P}_{i}\right)$, which equals to:

$$
e(P(i, n, \mu) / P(i, n-1, \mu)) d\left(P(i, n-1, \mu) / \bar{P}_{i}\right)+d(P(i, n, \mu) / P(i, n-1, \mu)),
$$

$n-r_{i}=e_{i}$ times to get $\delta_{i}$. Observe finally that $v\left(i, j-1, \mu, b_{j}\right)=0$, for all $1 \leq$ $j \leq n-e_{i}$. The last equality comes from the $E$-basis of Theorem 3 and the fact that: $-\Phi(i, j)=v\left(i, j-1, \mu, b_{j}\right)=v\left(i, j, \mu, y_{j}\right)$. Now, using the transitivity of the valuations, one can easily get that $v\left(i, n, \mu, y_{j}\right)=-\Phi(i, j) p^{n-j}$.

We are now ready to define the key-quantities for our Theorem. For $k=0,1, \ldots, p^{n}-1$, we define

$$
\nu_{i k}(m):=\left\lfloor\frac{m \delta_{i}-\sum_{j=1}^{n} a_{j}^{(k)} \Phi(i, j) p^{n-j}}{p^{e_{i}}}\right\rfloor,
$$

and $\Gamma_{k}(m):=\sum_{i=1}^{s} \nu_{i k}(m)$. In subsection 2.2 we interpret these $\Gamma_{k}(m)$ 's as the Boseck invariants.

Theorem 6. Let $G$ be a cyclic group of automorphisms of $F$, with $|G|=p^{n}$. Set $E=F^{G}$ and let $g_{E}$ be the genus of $E$. Let $m$ be a natural number with $m>1$. The regular representation of $G$ occurs $d_{p^{n}}=\Gamma_{p^{n}-1}(m)+\left(g_{E}-1\right)(2 m-1)$ times in the representation of $G$ on $\Omega_{F}(m)$. For $k=1, \ldots, p^{n}-1$, the indecomposable representation of degree $k$ occurs $d_{k}=\Gamma_{k-1}(m)-\Gamma_{k}(m)$ times.

Proof. As we saw, we have to compute $\operatorname{dim}_{K}\left(\Omega_{F}^{k+1}(m) / \Omega_{F}^{k}(m)\right)$, for $k=1, \ldots, p^{n}-1$. Choose an $x \in E$, such that $d x \neq 0$. Every holomorphic (poly)differential $\omega$ of $F$ can be written in a unique way as $\omega=\sum_{\nu=0}^{p^{n}-1} c_{\nu} w_{\nu}(d x)^{\otimes m}, c_{\nu} \in E$. 
We claim that

$$
(\sigma-1)^{k} \omega=0 \Rightarrow c_{k}=c_{k+1}=\cdots=c_{p^{n}-1}=0 .
$$

Proof of the claim. $(\sigma-1)^{k} \omega=(\sigma-1)^{k} \sum_{\nu=0}^{p^{n}-1} c_{\nu} w_{\nu}(d x)^{\otimes m}$. But $(\sigma-1)^{k}$ acts only on $w_{\nu}$ while leaving invariant the $c_{\nu}(d x)^{\otimes m}$. The $G$-action is given by $(\sigma-1)^{k} w_{\nu}=0$, for all $k>\nu$. Indeed $(\sigma-1)^{k+1} w_{k}=(\sigma-1)(\sigma-1)^{k} w_{k}=(\sigma-1) \prod_{\epsilon=1}^{n} \alpha_{\epsilon}^{(k)}$ ! and $\alpha_{\epsilon}^{(k)} ! \in K$, for all $\epsilon=1, \ldots, n$, so the product is fixed by the generator $\sigma$. From that we have

$$
\begin{aligned}
(\sigma-1)^{k} \omega & =(\sigma-1)^{k} \sum_{\nu=0}^{p^{n}-1} c_{\nu} w_{\nu}(d x)^{\otimes m} \\
& =\sum_{\nu \geq k}^{p^{n}-1} c_{\nu}(\sigma-1)^{k} w_{\nu}(d x)^{\otimes m} \\
& =c_{k}(d x)^{\otimes m} \prod_{\epsilon=1}^{n} \alpha_{\epsilon}^{(k)} !+\sum_{\nu \geq k+1}^{p^{n}-1} c_{\nu}(\sigma-1)^{k} w_{\nu}(d x)^{\otimes m}
\end{aligned}
$$

If the last equality is equal to zero, then we can see that

$$
\begin{aligned}
(\sigma-1)^{k+1} \omega & =(\sigma-1) c_{k}(d x)^{\otimes m} \prod_{\epsilon=1}^{n} \alpha_{\epsilon}^{(k)} !+\sum_{\nu \geq k+1}^{p^{n}-1} c_{\nu}(\sigma-1)^{k+1} w_{\nu}(d x)^{\otimes m} \\
& =\sum_{\nu \geq k+1}^{p^{n}-1} c_{\nu}(\sigma-1)^{k+1} w_{\nu}(d x)^{\otimes m}=0 .
\end{aligned}
$$

We apply the above argument recursively and we finally get

$$
c_{p^{n}-1}=0 \text {. }
$$

Now we write

$$
\omega=\sum_{\nu=0}^{p^{n}-2} c_{\nu}(\sigma-1)^{k} w_{\nu}(d x)^{\otimes m}
$$

and we repeat the whole procedure to get $c_{k}=c_{k+1}=\cdots=c_{p^{n}-2}=0$ and prove the claim.

We now have an alternative expression for the quotients of $\Omega_{F}^{i}(m)$. Namely $\operatorname{dim}_{K}\left(\Omega_{F}^{k+1}(m) / \Omega_{F}^{k}(m)\right)=$

(8) $\quad \operatorname{dim}_{K}\left\{c_{k} \in E:\right.$ there is an $\omega \in \Omega_{F}(m)$, with $\left.\omega=\sum_{\nu=0}^{k} c_{\nu} w_{\nu}(d x)^{\otimes m}\right\}$.

If $\omega=\sum_{\nu=0}^{k} c_{\nu} w_{\nu}(d x)^{\otimes m}$, then $(\sigma-1)^{k} \omega=\prod_{\epsilon=1}^{n} \alpha_{\epsilon}^{(k)} ! c_{k}(d x)^{\otimes m} \in \Omega_{F}(m)$, and from that we see that

$$
0 \leq v\left(i, n, \mu, \prod_{\epsilon=1}^{n} \alpha_{\epsilon}^{(k)} ! c_{k}(d x)^{\otimes m}\right)=v\left(i, n, \mu, c_{k}(d x)^{\otimes m}\right),
$$

thus $c_{k}(d x)^{\otimes m}$ is a $G$-invariant $m$-holomorphic differential on $F$. This means that

$$
\operatorname{div}_{F}\left(c_{k}(d x)^{\otimes m}\right)=\operatorname{Con}_{F / E}\left(\operatorname{div}_{E}\left(c_{k}(d x)^{\otimes m}\right)\right)+m \operatorname{Diff}(F / E)
$$

is an integral divisor. As the left side of the above equality has no poles, the same will be true for the right side, so the only poles that we allow for $c_{k}(d x)^{\otimes m}$, are the ones that can be canceled out from the factor $m \operatorname{Diff}(F / E)$. Hence $c_{k}(d x)^{\otimes m}$ can have poles only at ramified primes of $E$. As $\operatorname{div}(\omega)$ is an effective divisor, Lemma 4 requires that $v\left(i, n, \mu, c_{k}(d x)^{\otimes m} w_{k}\right) \geq 0$, for all $i, \mu$. So 
(9)

$$
v\left(i, n, \mu, w_{k}\right)+v\left(i, n, \mu, c_{k}(d x)^{\otimes m}\right) \geq 0 .
$$

We also have

$v\left(i, n, \mu, c_{k}(d x)^{\otimes m}\right)=v\left(i, n, \mu, \operatorname{Con}_{F / E} \operatorname{div}_{E}\left(c_{k}(d x)^{\otimes m}\right)\right)+v(i, n, \mu, m \operatorname{Diff}(F / E))$

$$
=p^{e_{i}} \bar{v}_{i}\left(c_{k}(d x)^{\otimes m}\right)+m \delta_{i} .
$$

Combining Eq. (9) and (10), we obtain $p^{e_{i}} \bar{v}_{i}\left(c_{k}(d x)^{\otimes m}\right)+m \delta_{i}+v\left(i, n, \mu, w_{k}\right) \geq 0$, or

$$
\bar{v}_{i}\left(c_{k}(d x)^{\otimes m}\right) \geq-\nu_{i k}(m) \text {. }
$$

The divisor of $E$

$$
D=\sum_{i=1}^{s} \nu_{i k}(m) \bar{P}_{i}+\operatorname{div}_{E}\left(c_{k}\right)+\operatorname{div}_{E}\left((d x)^{\otimes m}\right) \geq 0,
$$

is effective, which is equivalent to $c_{k} \in L\left(\sum_{i=1}^{s} \nu_{i k}(m) \bar{P}_{i}+\operatorname{div}_{E}\left((d x)^{\otimes m}\right)\right)$. Notice that the divisors $D$ and $\sum_{i=1}^{s} \nu_{i k}(m) \bar{P}_{i}+\operatorname{div}_{E}\left((d x)^{\otimes m}\right)$ are linear equivalent. Thus they have the same degrees and $\ell(D)=\ell\left(\sum_{i=1}^{s} \nu_{i k}(m) \bar{P}_{i}+\operatorname{div}_{E}\left((d x)^{\otimes m}\right)\right)$. With that in mind, we will use the Riemann-Roch Theorem on the function field $E$ in order to compute

$$
\ell(D):=\operatorname{dim}_{K} L\left(\sum_{i=1}^{s} \nu_{i k}(m) \bar{P}_{i}+\operatorname{div}_{E}\left((d x)^{\otimes m}\right)\right) .
$$

We have $\ell(m W)=\operatorname{deg}(m W)+1-g_{E}+\ell(W \backslash m W)$, where $W$ is a canonical divisor of $E$. It is well known that $\operatorname{deg} W=2 g_{E}-2, \ell(W)=g_{E}$ and if $\operatorname{deg}(A)<0$ then $\ell(A)=0$, for every divisor $A$ of $E$.

We have the following cases:

Case 1: $g_{E} \geq 2$.

Hence

$$
\operatorname{dim} \Omega_{E}(m)=\ell(m W)=m\left(2 g_{E}-2\right)+1-g_{E}+0
$$

and $\ell(m W)=(2 m-1)\left(g_{E}-1\right)$. Finally

$$
\ell\left(\sum_{i=1}^{s} \nu_{i k}(m) \bar{P}_{i}+\operatorname{div}_{E}\left((d x)^{\otimes m}\right)\right)=\operatorname{deg}\left(\sum_{i=1}^{s} \nu_{i k}(m) \bar{P}_{i}+\operatorname{div}_{E}\left((d x)^{\otimes m}\right)\right)+1-g_{E}+0,
$$

or equivalently

$$
\ell(D)=\Gamma_{k}(m)+\left(g_{E}-1\right)(2 m-1) .
$$

Case 2: $g_{E}=1$.

$\operatorname{deg}(W)=0$ and $\ell(m W)=1$, thus

$$
\ell(D)=\Gamma_{k}(m),
$$

because

$$
\ell(D)=\Gamma_{k}(m)+m\left(2 g_{E}-2\right)+1-g_{E}+\ell\left(W-m W-\Gamma_{k}(m)\right),
$$

with the last term of the sum being zero because

$$
\operatorname{deg}\left(W-m W-\Gamma_{k}(m)\right)=(1-m)\left(2 g_{E}-2\right)-\Gamma_{k}(m)=-\Gamma_{k}(m)<0 .
$$

Case 3: $g_{E}=0$.

In that case $\operatorname{deg}(W)=-2<0$, thus $\ell(m W)=0$, for all $m \geq 1$. Finally

$$
0 \leq \ell(D)=\Gamma_{k}(m)-2 m+1,
$$

because

$$
\operatorname{deg}(D)=\Gamma_{k}(m)-2 m \geq 0, \text { from Eq.111). }
$$

On the other hand as $c_{k} \in L\left(\sum_{i=1}^{s} \nu_{i k}(m) \bar{P}_{i}+\operatorname{div}_{E}\left((d x)^{\otimes m}\right)\right)$, from Eq. (8) we get, for all cases, that

$$
\operatorname{dim}_{K}\left(\Omega_{F}^{k+1}(m) / \Omega_{F}^{k}(m)\right) \leq \ell(D)=\Gamma_{k}(m)+\left(g_{E}-1\right)(2 m-1) .
$$


We want to show that inequality (13) is actually an equality, so using Eq. (4), we are going to calculate the $d_{k}{ }^{\prime} s$.

We compute:

$$
\begin{aligned}
(2 m-1)\left(g_{F}-1\right) & =\operatorname{dim}_{K} \Omega_{F}(m)=\sum_{k=0}^{p^{n}-1} \operatorname{dim}_{K}\left(\Omega_{F}^{k+1}(m) / \Omega_{F}^{k}(m)\right) \\
& \leq \sum_{k=0}^{p^{n}-1} \Gamma_{k}(m)+\sum_{k=0}^{p^{n}-1}\left(g_{E}-1\right)(2 m-1) \\
& =\sum_{k=0}^{p^{n}-1} \Gamma_{k}(m)+p^{n}(2 m-1)\left(g_{E}-1\right) .
\end{aligned}
$$

If we show that $\operatorname{dim}_{K} \Omega_{F}(m)$ is equal to Eq. 14, then we have the desired equality. This is done using Riemann-Hurwitz Theorem:

$$
2 g_{F}-2=\frac{[F: E]}{[K: K]}\left(2 g_{E}-2\right)+\operatorname{deg} \operatorname{Diff}(F / E)
$$

where $\operatorname{Diff}(F / E)=\sum_{i=1}^{s} \sum_{P(i, n, \mu) / \bar{P}_{i}} \delta_{i} \cdot P(i, n, \mu)$. Since $K$ is algebraically closed, then $\operatorname{deg} P(i, n, \mu)=f\left(P(i, n, \mu) / \bar{P}_{i}\right)=\left[\mathscr{O}_{P(i, n, \mu)} / P(i, n, \mu): \mathscr{O}_{\bar{P}_{i}} / \bar{P}_{i}\right]=1$, for all $i$. Moreover, since the $F / E$ is Galois the number of places of $F$ above $\bar{P}_{i}$ is $\mu$, where $[F: E]=e\left(P(i, n, \mu) / \bar{P}_{i}\right) \cdot f\left(P(i, n, \mu) / \bar{P}_{i}\right) \cdot \mu$, so $\mu=p^{n-e_{i}}$. We can now calculate

$$
(2 m-1)\left(g_{F}-1\right)=p^{n}\left(g_{E}-1\right)(2 m-1)+\frac{1}{2}(2 m-1) \sum_{i=1}^{s} p^{n-e_{i}} \delta_{i} .
$$

From Eq. 14 and (15), it is enough to show that

$$
\sum_{k=0}^{p^{n}-1} \nu_{i k}(m)=\frac{1}{2}(2 m-1) p^{n-e_{i}} \delta_{i}, \text { for all } i
$$

Remark 7. Observe from Eq. (16) that it is enough to show that

$$
\frac{2}{2 m-1} \sum_{k=0}^{p^{n}-1} \Gamma_{k}(m)=\operatorname{deg} \operatorname{Diff}(F / E)
$$

We compute:

$$
\begin{aligned}
\sum_{k=0}^{p^{n}-1} \nu_{i k}(m) & =\sum_{k=0}^{p^{n}-1}\left\lfloor\frac{m \delta_{i}-\sum_{j=1}^{n} a_{j}^{(k)} \Phi(i, j) p^{n-j}}{p^{e_{i}}}\right\rfloor \\
& =p^{n-e_{i}} m \delta_{i}-\frac{1}{p^{e_{i}}} \sum_{k=0}^{p^{n}-1} \sum_{j=1}^{n} a_{j}^{(k)} \Phi(i, j) p^{n-j} \\
& -\sum_{k=0}^{p^{n}-1}\left\langle\frac{m \delta_{i}-\sum_{j=1}^{n} a_{j}^{(k)} \Phi(i, j) p^{n-j}}{p^{e_{i}}}\right\rangle .
\end{aligned}
$$


First we take care of the second summation. Fix a $j$. As $k$ runs over $0, \ldots, p^{n}-1$, the elements $a_{j}^{(k)}$ take all the values from zero to $p-1, p^{n-1}$ times. Considering this we have

$$
\begin{aligned}
\frac{1}{p^{e_{i}}} \sum_{k=0}^{p^{n}-1} \sum_{j=1}^{n} a_{j}^{(k)} \Phi(i, j) p^{n-j} & =\frac{1}{p^{e_{i}}} \sum_{j=1}^{n} \Phi(i, j) p^{n-j} \sum_{k=0}^{p^{n}-1} a_{j}^{(k)} \\
& =\frac{1}{p^{e_{i}}} \sum_{j=1}^{n} \Phi(i, j) p^{n-j} p^{n-1} \sum_{k=0}^{p-1} k \\
& =\frac{1}{2} p^{n-1} p(p-1) \frac{1}{p^{e_{i}}} \sum_{j=1}^{n} \Phi(i, j) p^{n-j} \\
& =\frac{p^{n-e_{i}}}{2}(p-1) \sum_{j=1}^{n} \Phi(i, j) p^{n-j} \\
& =\frac{p^{n-e_{i}}}{2}\left(\delta_{i}+1-p^{e_{i}}\right),
\end{aligned}
$$

where the last equality came from Lemma 5 .

Then we consider the fractional part. Observe that $\Phi(i, j)=0$, for all $j \leq n-e_{i}$ and $\Phi(i, j)$ is relatively prime to $p$, from the standard form hypothesis. We notice that as $a_{j}^{(k)}$ 's runs over $0, \ldots, p-1$ for $j \geq n-e_{i}+1$, the numbers $\sum_{j=n-e_{i}+1}^{n} a_{j}^{(k)} p^{n-j}$, form a complete system $\bmod p^{e_{i}}$. In the case where $r=0$, then the same numbers, for $j \geq 1$, form a complete system $\bmod p^{n}$. It is well known from elementary number theory that the same is true for $\sum_{j=n-e_{i}+1}^{n} a_{j}^{(k)} \Phi(i, j) p^{n-j}$, using the fact that $\operatorname{g.c} . \mathrm{d}(\Phi(i, j), p)=1$. Thus as $k$ runs over $0, \ldots, p^{n}-1$, the numbers $m \delta_{i}-\sum_{j=1}^{n} a_{j}^{(k)} \Phi(i, j) p^{n-j}$ run over a complete residue system $\bmod p^{e_{i}}$ (in fact $z \pm \sum_{j=1}^{n} a_{j}^{(k)} \Phi(i, j) p^{n-j}$ run through a complete residue system for all $z \in \mathbb{Z}), p^{n-e_{i}}$ times. We are ready to calculate

$$
\begin{aligned}
\sum_{k=0}^{p^{n}-1}\left\langle\frac{m \delta_{i}-\sum_{j=1}^{n} a_{j}^{(k)} \Phi(i, j) p^{n-j}}{p^{e_{i}}}\right\rangle & =\frac{p^{n-e_{i}}}{p^{e_{i}}} \sum_{k=0}^{p^{e_{i}}-1} k \\
& =\frac{p^{n-e_{i}}}{p^{e_{i}}} \frac{p^{e_{i}}\left(p^{e_{i}}-1\right)}{2} \\
& =\frac{1}{2}\left(p^{n}-p^{n-e_{i}}\right) .
\end{aligned}
$$

The final step is to combine the Equations (17), 18) and the first summand, in order to obtain

$$
\begin{aligned}
\sum_{k=0}^{p^{n}-1} \nu_{i k}(m) & =p^{n-e_{i}} m \delta_{i}-\frac{p^{n-e_{i}}}{2}\left(\delta_{i}+1-p^{e_{i}}\right)-\frac{1}{2}\left(p^{n}-p^{n-e_{i}}\right) \\
& =\frac{2 p^{n-e_{i}} m \delta_{i}-p^{n-e_{i}} \delta_{i}-p^{n-e_{i}}+p^{n}-p^{n}+p^{n-e_{i}}}{2} \\
& =\frac{1}{2}\left(p^{n-e_{i}} \delta_{i}(2 m-1)\right),
\end{aligned}
$$

We showed that inequality (14) is actually an equality. Using Eq. (4), to compute the $d_{k}$ 's, Theorem follows.

Remark 8 (The case $m=1, r=0$ ). If $g_{E} \geq 2$ then $\ell(D)=\Gamma_{k}(1)+\left(g_{E}-1\right)$, for all $0 \leq k<p^{n}-1$ and $\ell(D)=\left(g_{E}-1\right)+1$ for $k=p^{n}-1$. If $g_{E}=1$, then $\ell(D)=\Gamma_{k}(1)$, for $0 \leq k \leq p^{n}-2$, while $\ell(D)=1$ for $k=p^{n}-1$. Finally if $g_{E}=0$ then $\ell(D)=\Gamma_{k}(1)-1$, 
for $0 \leq k \leq p^{n}-2$, while $\ell(D)=0$ for $k=p^{n}-1$. The extra cases that we do not consider in Eq. (13) are

$$
\begin{aligned}
& \text { for } g_{E} \geq 2, d_{p^{n}}=\operatorname{dim}_{K}\left(\Omega_{F}^{p^{n}}(1) / \Omega_{F}^{p^{n}-1}(1)\right) \leq \ell(D)=g_{E}, \\
& \text { for } g_{E}=1, d_{p^{n}}=\operatorname{dim}_{K}\left(\Omega_{F}^{p^{n}}(1) / \Omega_{F}^{p^{n}-1}(1)\right) \leq \ell(D)=1, \\
& \text { for } g_{E}=0, d_{p^{n}}=\operatorname{dim}_{K}\left(\Omega_{F}^{p^{n}}(1) / \Omega_{F}^{p^{n}-1}(1)\right) \leq \ell(D)=0 .
\end{aligned}
$$

Observe that for the exceptional cases- the inequalities above-, we have that

$$
\begin{aligned}
g_{F} & =\operatorname{dim}_{K} \Omega_{F}(1)=\sum_{k=0}^{p^{n}-1} \operatorname{dim}_{K}\left(\Omega_{F}^{k+1}(m) / \Omega_{F}^{k}(m)\right) \\
& \leq \sum_{k=0}^{p^{n}-1} \Gamma_{k}(1)+\left(p^{n}\left(g_{E}-1\right)+1\right) .
\end{aligned}
$$

Thus, in order to prove that these inequalities are equalities, with the help of Eq. 115 we just have to show Eq. (16) for $m=1$.

This is a way to arrive at [23, Theorem 2], when a place in $\mathbb{P}_{E}$ is totally ramified in $F$, or equivalently when there is not an unramified subextension of $F / E$ (i.e. $r=0$ ), in order to prove an analogous result for the $m=1$ case:

Theorem 9 (Valentini-Madan). For $m=1$ and when exists a place in $\mathbb{P}_{E}$ that is totally ramified in $F$, the regular representation of $G$ occurs $d_{p^{n}}=g_{E}$ times in the representation of $G$ on $\Omega_{F}(1)$. For $k=1, \ldots, p^{n}-1$, the indecomposable representation of degree $k$ occurs $d_{k}=\Gamma_{k-1}(1)-\Gamma_{k}(1)+\beta$ times, where $\beta$ equals to -1 , if $k=p^{n}-1$ and equals to zero otherwise.

As Valentini and Madan observed for the case $m=1, \Gamma_{k}(1)=0$, if $\nu_{i k}(1)=0$ for all $i$ 's, and that could happen if and only if $k \geq p^{n}-p^{r}$. However when $m \geq 2$ then $\Gamma_{k}(m) \supsetneqq 0$. Thus for the $m=1$ case one needs to distinguish cases on whether Boseck's invariants are zero or not, in order to derive a result for the $K[G]$ module structure of $\Omega_{F}(1)$ which will be depended on $r$. If we assume that $r \neq 0$ in the case $m=1$ then one should use Tamagawa or Valentini results, [20] [22], in order to treat the unramified $E_{r} / E$ extension (see [23, proof of Theorem 2 , case $\left.k \geq p^{n}-p^{r}\right]$ ).

This is the major difference between the exposition found there and the one followed here, which also shows that for $m \gtrless 1$ Boseck invariants, and hence the conditions for holomorphicity that they compactly express, as well as the $K[G]$ module structure of $\Omega_{F}(m)$, do not depend on $r$ and thus on the existence of an unramified subextension, $E_{r} / E$, that appears when no place is totally ramified in $F / E$.

2.2. A New Basis for Holomorphic Differentials. We now proceed to a basis construction for the holomorphic $m$-(poly)differentials $\Omega_{F}(m)$ when $E$ is rational. Without loss of generality, we will assume that $E=K(x)$ and $F / K(x)$ will be a cyclic extension of degree $p^{n}$. The main result of this subsection, looks like Lemma 5 of Madden [16]. The main difference is that there, he assumed that the infinite prime of E ramifies and takes two cases; case one stands for the $P(i, n, \mu)$ 's that are lying over the "finite" primes of $E$ while case two, for $P(i, n, \mu)$ 's that are lying over the "infinite" prime of $E, p_{\infty}$.

Here we assume that the infinite prime of E does not ramify and give a single basis for the holomorphic differentials first $(m=1)$, and then for holomorphic $m$-(poly)differentials, $(m>1)$.

The quantities that we defined at Eq. (7) play a major role for us. They are the quantities that Valentini and Madan defined at [23, p.110] and the quantities that followed from the restrictions on $\nu$, at Lemma 5 of Madden, [16]. These are in fact, the same quantities that Boseck constructed in his work [3] in the late fifties, which led him to a similar basis for 
$\Omega_{F}(1)$, when $F / K(x)$ was an Artin-Schreier or a Kummer (of degree $q$, with g.c.d $(p, q)=$ $1)$ extension, of an algebraic function field. Let us have a closer analysis.

$$
\operatorname{div}_{F}\left((d x)^{\otimes m}\right)=m \operatorname{Diff}(F / K(x))-2 m(x)_{\infty},
$$

or using Lemma[5] we have

$$
\begin{gathered}
m \sum_{i=1}^{s} p^{n-e_{i}}\left(\sum_{j=1}^{n}(p-1) \Phi(i, j) p^{n-j}+\left(p^{e_{i}}-1\right)\right) \cdot P(i, n, \mu)-2 m \operatorname{Con}_{F / K(x)}\left(p_{\infty}\right)= \\
=m \sum_{i=1}^{s} p^{n-e_{i}} \delta_{i} \cdot P(i, n, \mu)-2 m \operatorname{Con}_{F / K(x)}\left(p_{\infty}\right) .
\end{gathered}
$$

Using Lemma 5 and observing that $v\left(i, n, \mu, w_{k}\right)=v\left(i, n, \mu^{\prime}, w_{k}\right)$, we can write:

$$
\operatorname{div}_{F}\left(w_{k}\right)=P_{w_{k}}-\sum_{i=1}^{s} p^{n-e_{i}} \sum_{j=1}^{n} a_{j}^{(k)} \Phi(i, j) p^{n-j} \cdot P(i, n, \mu)
$$

where $P_{w_{k}}$ is an effective divisor of $F$, prime to $P(i, n, \mu)$, for all $i$ 's. Then

$\operatorname{div}_{F}\left(w_{k}(d x)^{\otimes m}\right)=P_{w_{k}}+\sum_{i=1}^{s} p^{n-e_{i}}\left(m \delta_{i}-\sum_{j=1}^{n} a_{j}^{(k)} \Phi(i, j) p^{n-j}\right) \cdot P(i, n, \mu)-2 m \operatorname{Con}_{F / K(x)}\left(p_{\infty}\right)$

We analyze $p^{n-e_{i}}\left(m \delta_{i}-\sum_{j=1}^{n} a_{j}^{(k)} \Phi(i, j) p^{n-j}\right) \bmod p^{n}$ :

$p^{n-e_{i}}\left(m \delta_{i}-\sum_{j=1}^{n} a_{j}^{(k)} \Phi(i, j) p^{n-j}\right)=\nu_{i k}(m) \cdot p^{n}+\rho_{i}^{(k, m)}$, with $0 \leq \rho_{i}^{(k, m)} \leq p^{n}-1$.

Notice that we are consistent with the definition of $\nu_{i k}(m)$ 's given in Eq. (7). We denote the ramified places of E, $\bar{P}_{i}, i=1, \ldots, s$ with $\bar{P}_{i}(x)$, (since $E$ is rational then every ramified place of $E, \bar{P}_{i}$, corresponds to an irreducible polynomial, $\left.\bar{P}_{i}(x)\right)$ and let

$$
g_{k}(x)=\prod_{i=1}^{s} \bar{P}_{i}(x)^{\nu_{i k}(m)}
$$

Then the quantity $\Gamma_{k}(m)$ of Eq. (7) is naturally defined as the degree of $g_{k}(x)$. This is the Boseck invariant for this case. We then have

$\operatorname{div}_{F}\left(w_{k}\left[g_{k}(x)\right]^{-1}(d x)^{\otimes m}\right)=\sum_{i=1}^{s} \rho_{i}^{(k, m)} \cdot P(i, n, \mu)+\left(\Gamma_{k}(m)-2 m\right) \operatorname{Con}_{F / K(x)}\left(p_{\infty}\right)+P_{w_{k}}$.

Observe that if

$$
\Gamma_{k}(m) \geq 2 m
$$

then, the divisor of Eq. 20 is effective, so for $k=0 \ldots, p^{n}-1, \Gamma_{k}(m) \geq 2 m$ and for $0 \leq \nu \leq \Gamma_{k}(m)-2 m$, the $x^{\nu} w_{k}\left[g_{k}(x)\right]^{-1}(d x)^{\otimes m}$ is a holomorphic $m$-(poly)differential of $F$.

Recall that the set $w_{k}=y_{1}^{a_{1}^{(k)}} y_{2}^{a_{2}^{(k)}} \cdots y_{n}^{a_{n}^{(k)}}, 0 \leq k \leq p^{n}-1$ is an $E$-basis of $F$. The main result of this subsection is:

Lemma 10. The set $\left\langle x^{\nu} w_{k}\left[g_{k}(x)\right]^{-1}(d x)^{\otimes m}: 0 \leq k \leq p^{n}-1+\beta_{m}, 0 \leq \nu \leq \Gamma_{k}(m)-\right.$ $2 m, m \geq 1\rangle$, is a $K$-basis for the space of the $m$-(poly)differentials $\Omega_{F}(m)$, with $\beta_{1}=-1$, if $m=1$ and $\beta_{m}=0$ if $m \geq 2$. 
Proof. First remember that

$$
\nu_{i k}(m):=\left\lfloor\frac{m \delta_{i}-\sum_{j=1}^{n} a_{j}^{(k)} \Phi(i, j) p^{n-j}}{p^{e_{i}}}\right\rfloor .
$$

We now observe that:

Since the base field is rational, $r=0$, because every finite separable extension of the rational function field should be ramified. Thus, for $m=1$, the condition $a_{j}^{(k)}=p-1$, for all $j=1, \ldots n$, is equivalent to $k=p^{n}-1$ and $\Gamma_{p^{n}-1}(1)=0$, while

for $m>1$, we always have that $\Gamma_{k}(m) \neq 0$ for every $k$.

It is enough to prove the equalities below:

(i) For $m=1$, we must prove that $\sum_{k=0}^{p^{n}-2}\left(\Gamma_{k}(1)-1\right)=\operatorname{dim}_{K} \Omega_{F}(1)=g_{F}$,

(ii) For $m \geq 2$, we must see that $\sum_{k=0}^{p^{n}-1}\left(\Gamma_{k}(m)-2 m+1\right)=\operatorname{dim}_{K} \Omega_{F}(m)=$ $(2 m-1)\left(g_{F}-1\right)$. This number is well defined since $g_{F} \geq 2$ from our hypothesis.

For case (i), we see from Eq. 19] for $m=1$ that this number has been computed :

$$
\sum_{k=0}^{p^{n}-1} \nu_{i k}(m)=\frac{1}{2}\left(p^{n-e_{i}} \delta_{i}\right)
$$

So

$$
\sum_{k=0}^{p^{n}-2}\left(\Gamma_{k}(1)-1\right)=\frac{1}{2} \sum_{i=1}^{s}\left(p^{n-e_{i}} \delta_{i}\right)-p^{n}+1=g_{F} .
$$

Where the last equality comes from Riemann-Hurwitz Theorem, a version of that can be found in Eq. (15). For $m \geq 2$ Eq. (19), gives that

$$
\begin{aligned}
\sum_{k=0}^{p^{n}-1} \nu_{i k}(m) & =\frac{1}{2}\left(p^{n-e_{i}} \delta_{i}(2 m-1)\right), \text { thus } \\
\sum_{k=0}^{p^{n}-1}\left(\Gamma_{k}(m)-2 m+1\right) & =\sum_{i=1}^{s}\left(\frac{1}{2} p^{n-e_{i}} \delta_{i}(2 m-1)\right)-p^{n}(2 m-1) \\
& =(2 m-1)\left(\frac{1}{2} \sum_{i=1}^{s} p^{n-e_{i}} \delta_{i}-p^{n}\right) \\
\text { Eq. } & \left(g_{F}-1\right)(2 m-1) .
\end{aligned}
$$

The following Remarks are here to confirm the correctness of our arguments, given in the proof of the Theorem 6 in subsection 2.1

Remark 11. Observe that the restriction for $\Gamma_{k}(m)$, given in Eq. (12), when the genus of $E$ was zero, is the same restriction that results from our basis in order to ensure that an m-(poly)differential is holomorphic (see Eq. (21)).

Remark 12. A second, simpler proof for Theorem 6 can be given when $g_{E}=0$, using the basis of Lemma 10. Indeed having Eq. (8) instead of using Riemann-Roch Theorem to count the $c_{k}$ 's, we use our basis. From Eq. (8) the needed differentials are of the form $\omega=x^{k}\left[g_{k}(x)\right]^{-1} w_{k}(d x)^{\otimes m}$ with $c_{k}:=x^{k}\left[g_{k}(x)\right]^{-1}$ and there are $\Gamma_{k}(m)-2 m+1$ of them, for every $k=1, \ldots, p^{n}-1$. So for $k=1, \ldots, p^{n}-1$, with the help of Eq. (4), we get that $d_{k}=\Gamma_{k-1}(m)-2 m+1-\left(\Gamma_{k}(m)-2 m+1\right)=\Gamma_{k-1}(m)-\Gamma_{k}(m)$, while for $k=p^{n}$ we have that $d_{p^{n}}=\Gamma_{p^{n}-1}(m)-2 m+1$. 
2.3. A classical theorem of Hurwitz. We will study now how Boseck invariants behave when $F / E$ is a cyclic tame ramified extension of function fields. Let $F / E$ is be a cyclic Kummer extension of degree $n$, with g.c.d $(n, p)=1$. Choose a primitive $n$th root of unity, $\zeta$ of $K$. Let $F=E(y)$ and

$$
y^{n}=u, u \in E .
$$

Let $\bar{P}_{i} \in \mathbb{P}_{E}$, with $1 \leq i \leq r$, be the ramified places of $E$ in $F$ and $P_{i} \in \mathbb{P}_{F}$ the places above $\bar{P}_{i}$. We can assume that if $\sigma$ is a generator of $G$ then $\sigma(y)=\zeta y$ and also that $0 \supsetneqq v_{\bar{P}_{i}}(u) \supsetneqq n$. Using Kummer theory [19, p.110, Proposition III.7.3] we have that $e_{i}=e\left(P_{i} / \bar{P}_{i}\right)=\frac{n}{\text { g.c.d. }\left(n, v_{\bar{P}_{i}}(u)\right)}$ and $\delta_{i}=e_{i}-1$. Finally, set $\Phi(i)=v_{P_{i}}(y)=\frac{e_{i} v_{\bar{P}_{i}}(u)}{n}$.

If we define $\Omega_{F}^{k}(1)=\left\{\omega \in \Omega_{F}(1) \mid \sigma(\omega)=\zeta^{k} \omega\right\}$, for $0 \leq k \leq n-1$, then

$$
\Omega_{F}(1)=\bigoplus_{k=0}^{n-1} \Omega_{F}^{k}(1),
$$

and the corresponding $d_{k}$ 's for this case are $d_{k}=\operatorname{dim}_{K} \Omega_{F}^{k}(1)$. It is also well known (see at [6, p.272, V.2.2, Corollary]) that $d_{0}=\operatorname{dim}_{K} \Omega_{F}^{G}(1)=g_{E}$. Notice that this is false in positive characteristic in the case of wild ramification.

In order to find the $K[G]$ module structure of $\Omega_{F}(1)$, we should compute once more the $d_{k}$ 's. For that reason we should find the Boseck invariant for this case, i.e. consider the corresponding rational extension. If $E=K(x)$, then Boseck [3, p. 50, Satz 16], proved that (for $m=1$ ) :

Proposition 13 (Boseck). The set $\left\langle x^{\nu} y^{-k} g_{k}(x) d x: 0 \supsetneqq k \leq n-1,0 \leq \nu \leq \Gamma_{k}(1)-2\right\rangle$ is a K-basis for the space of $\Omega_{F}(1)$, where $g_{k}(x)=\prod_{i=1}^{r} \bar{P}_{i}(x)^{\left\lfloor\frac{k \Phi(i)}{e_{i}}\right\rfloor}$ and

$$
\Gamma_{k}(1)=\sum_{i=1}^{r}\left\langle\frac{k \Phi(i)}{e_{i}}\right\rangle .
$$

Remark 14. One crucial step in the proof of Proposition 13 is to show that

$$
2 \sum_{k=1}^{n-1} \Gamma_{k}(1)=\operatorname{deg} \operatorname{Diff}(F / E) .
$$

We have $k$ distinct irreducible representations of degree 1 . If $g_{E}=0$ and $k \neq 0$, we may count the differentials in Proposition 13 in order to find the $d_{k}$ 's. We see that $\omega \in \Omega_{F}^{k}(1)$ if $\omega=x^{\nu} y^{n-k} g_{n-k}(x) d x$. Their number equals to

$$
\begin{aligned}
\Gamma_{n-k}(1)-1 & =\sum_{i=1}^{r}\left\langle\frac{n-k \Phi(i)}{e_{i}}\right\rangle-1 \\
& =\sum_{i=1}^{r}\left\langle\frac{-k \Phi(i)}{e_{i}}\right\rangle-1 .
\end{aligned}
$$

So the $k$ th representation occurs $d_{n-k}=\Gamma_{n-k}(1)-1$ times in the representation of $G$ in $\Omega_{F}(1)$. When $k=0$ we know that occurs $d_{0}=g_{E}=0$ times.

Notice that, in general, the $\Gamma_{k}(m)$ 's depend on the different exponent and the evaluation of basis elements, thus the genus of the base field $E, g_{E}$ does not affect them. We can claim now that the same result will be true when $g_{E} \geq 0$. Indeed this is the Hurwitz theorem (compare also to [23, Theorem 2]):

Theorem 15 (Hurwitz). For $k=0, \ldots, n-1$, we have $n$ distinct irreducible representations of degree 1 . The $k$ th representation occurs $d_{n-k}:=\Gamma_{n-k}(1)-1+g_{E}$ times in the representation of $G$ in $\Omega_{F}(1)$, when $k \neq 0$ and $g_{E}$ times when $k=0$. 


\section{The Elementary Abelian Case}

Let $K$ be an algebraically closed field of characteristic $p$ and consider an elementary abelian Galois extension $F / K(x)$ of the rational function field $E=K(x)$. Set $G=$ $\operatorname{Gal}(F / K(x))$ and assume that $|G|=p^{n}$. We also assume that every place of $K(x)$ that is ramified in the above extension is totally ramified, i.e, $F=K(x, y)$, where $y$ satisfies the equation:

$$
y^{q}-y=\frac{g(x)}{\left(x-a_{1}\right)^{\Phi(1)} \cdots\left(x-a_{r}\right)^{\Phi(r)}},
$$

where $q=p^{n}, n \geq 1, g(x) \in K[x]$, and $\operatorname{deg} g(x) \leq \sum_{i=1}^{r} \Phi(i)=M, g\left(a_{i}\right) \neq 0$. Thus, $p_{\infty}$, the infinite place of $K(x)$, is assumed to be unramified in $F$. Finally all the $\Phi(i)$ 's are relatively prime to $p$ for all $i=1 \ldots, r$. Let $p_{i} \in \mathbb{P}_{K(x)}, i=1, \ldots, r$, be the rational places of $K(x)$ that are totally ramified in $F$, corresponding to $\left(x-a_{i}\right), a_{i} \in K$.

First we fix some notation, similar to the notation given in subsection 2.2 It is well known (see for example Stichtenoth, [19, Prop. III.7.10]) that $\operatorname{Con}_{F / K(x)}\left(p_{i}\right)=$ $p^{n} P_{i}$, for $P_{i} \in \mathbb{P}_{F}$ above $p_{i}$ and $\operatorname{Diff}(F / K(x))=\sum_{i=1}^{r}\left(p^{n}-1\right)(\Phi(i)+1) P_{i}$, for all $i=1, \ldots, r$. We also know that for any $\beta \in \mathbb{F}_{q}$ the element $\sigma_{\beta} \in G$ acts on the generator of the extension $y$ as: $\sigma_{\beta}(y)=y+\beta$. For an $m$-(poly)differential of $F,(d x)^{\otimes m}$ we will also have

$$
\begin{aligned}
\operatorname{div}_{F}\left((d x)^{\otimes m}\right) & =m \operatorname{Diff}(F / K(x))-2 m(x)_{\infty} \\
& =m \sum_{i=1}^{r}\left(p^{n}-1\right)(\Phi(i)+1) P_{i}-2 m \operatorname{Con}_{F / K(x)}\left(p_{\infty}\right),
\end{aligned}
$$

where $(x)_{\infty}$ is the pole divisor of $x$. Taking degrees to the above divisors (or alternatively using the Riemann-Hurwitz formula), we have

$$
g_{F}=\frac{p^{n}-1}{2}\left(-2+\sum_{i=1}^{r}(\Phi(i)+1)\right) .
$$

Set $\operatorname{div}_{F}(y)=P_{y}-\sum_{i=1}^{r} \Phi(i) P_{i}$, for an effective divisor $P_{y}$ of $F$, prime to $P_{i}$, for $i=1, \ldots, r$. Then for $k=0, \ldots, p^{n}-1$ we compute $\operatorname{div}_{F}\left(y^{k}(d x)^{\otimes m}\right)$, which equals to

$$
\begin{aligned}
& =\operatorname{div}_{F}\left(y^{k}\right)+\operatorname{div}_{F}\left((d x)^{\otimes m}\right) \\
& =k P_{y}-k \sum_{i=1}^{r} \Phi(i) P_{i}+m \sum_{i=1}^{r}\left(p^{n}-1\right)(\Phi(i)+1) P_{i}-2 m \operatorname{Con}_{F / K(x)}\left(p_{\infty}\right) \\
(26) & =\sum_{i=1}^{r}\left(\left(\left(p^{n}-1\right) m-k\right) \Phi(i)+m\left(p^{n}-1\right)\right) P_{i}+k P_{y}-2 m \operatorname{Con}_{F / K(x)}\left(p_{\infty}\right) .
\end{aligned}
$$

Then we consider $\left(\left(p^{n}-1\right) m-k\right) \Phi(i)+m\left(p^{n}-1\right)$ modulo $p^{n}$

$$
\left(\left(p^{n}-1\right) m-k\right) \Phi(i)+m\left(p^{n}-1\right)=\nu_{i k}(m) \cdot p^{n}+\rho_{i}^{(k, m)},
$$

where $0 \leq \rho_{i}^{(k, m)} \leq p^{n}-1$, is the remainder of the division, so

$$
\nu_{i k}(m)=\left\lfloor\frac{m\left(p^{n}-1\right)(\Phi(i)+1)-k \Phi(i)}{p^{n}}\right\rfloor .
$$

We also take

$$
g_{k}(x):=\prod_{i=1}^{r}\left(x-a_{i}\right)^{\nu_{i k}(m)},
$$

and let $\Gamma_{k}(m)=\sum_{i=1}^{r} \nu_{i k}(m)$, be the Boseck invariant for this case.

Observe from Eq. (27) that for $m=1$, if $\Gamma_{k}(1)=0$, then $\nu_{i k}(1)=0$ for all $i=1, \ldots, r$ and that could happen only when $k=p^{n}-1$ since $\nu_{i k}(1) \geq 0$. 
For $m>1$, this is not the case because $\nu_{i k}(m)>0$, for all $k$ 's. We finally observe that $\operatorname{div}_{F}\left[g_{k}(x)\right]^{-1} y^{k}(d x)^{\otimes m}$ is an effective divisor if

$$
\Gamma_{k}(m)=\sum_{i=1}^{r} \nu_{i k}(m) \geq 2 m
$$

because

$$
\operatorname{div}_{F}\left(y^{k}\left[g_{k}(x)\right]^{-1}(d x)^{\otimes m}\right)=\sum_{i=1}^{r} \rho_{i}^{(k, m)} P_{i}+k P_{y}+\left(\sum_{i=1}^{r} \nu_{i k}(m)-2 m\right) \operatorname{Con}_{F / K(x)}\left(p_{\infty}\right) .
$$

So when inequality 29] is fulfilled, then $x^{\nu} y^{k}\left[g_{k}(x)\right]^{-1}(d x)^{\otimes m}$ is a holomorphic $m$ (poly)differential, for $0 \leq k \leq p^{n}-1,0 \leq \nu \leq \Gamma_{k}(m)-2 m$ and $m \geq 1$.

Remark 16. We kept the notation of this section as close is possible, to the notation used in Section 2. Generally the quantities $\nu_{i k}(m)$, for a p-extension with $p \mid$ charK, are equal to

$$
\left\lfloor\frac{m \delta_{i}+\{\text { evaluation of the } k t h E \text { basis element of } F \text { by a normalized valuation of } F\}}{p^{e_{i}}}\right\rfloor,
$$

where $i$ runs the ramified places of $E, \delta_{i}$ is the different exponent of the extension and $e_{i}$ the ramification indices of the ramified primes of $E$ in $F$. The basis element is evaluated by a (normalized) valuation determined by a place of $F$ above a ramified place of $E$. Here we have total ramification, so $e_{i}=n$. In the case where $F / E$ was a cyclic extension, the term in the brackets is nothing else than $v\left(i, n, \mu, w_{k}\right)=-\sum_{j=1}^{n} a_{j}^{(k)} \Phi(i, j) p^{n-j}$ given in Lemma 5 . Here if we take the standard $E$ basis for $F$, namely, $\left\{w_{k}:=y^{k} \mid 0 \leq\right.$ $\left.k \leq p^{n}-1,\right\}_{E}$, we will then have that $v_{P_{i}}\left(w_{k}\right)=k v_{P_{i}}(y)$. Letting the right hand of $E q$. (23) be equal to an $u \in E$, then from the strict triangle inequality [19. p.5, Lemma I.1.10] we have that $v_{p_{i}}(u)=\min \left\{v_{p_{i}}\left(y^{q}\right), v_{p_{i}}(y)\right\}=q v_{p_{i}}(y)=v_{P_{i}}(y)$. Letting now $-\Phi(i)=v_{p_{i}}(u)$ the Remark follows. Finally observe that $\Phi(i, j)=\Phi(i)$ because we haven't got a cyclic extension, thus we cannot get an analogous field tower like we had in Eq. (5). That, explains the independence from $j$.

3.1. Basis Construction. Following Boseck (see [3], Satz 15), we now prove the analogue of Lemma 10.

Proposition 17. The set $\Sigma_{m}:=\left\langle x^{\nu} y^{k}\left[g_{k}(x)\right]^{-1}(d x)^{\otimes m}: 0 \leq k \leq p^{n}-1+\beta_{m}, 0 \leq\right.$ $\left.\nu \leq \Gamma_{k}(m)-2 m, m \geq 1\right\rangle$ is a K-basis for the space of the $m$-(poly)differentials $\Omega_{F}(m)$, with $\beta_{1}=-1$, if $m=1$ and $\beta_{m}=0$ if $m \geq 2$.

Proof. For $m=1$, we have nothing to prove since this is Theorem 2 of Garcia [8]. For $m>1$ it is enough to show that $\sum_{k=0}^{p^{n}-1}\left(\Gamma_{k}(m)-2 m+1\right)=\operatorname{dim}_{K} \Omega_{F}(m)$.

First, we will compute $\sum_{k=0}^{p^{n}-1} \nu_{i k}(m)$. This is equal to

$$
\begin{aligned}
& =\sum_{k=0}^{p^{n}-1} \frac{m\left(p^{n}-1\right)(\Phi(i)+1)-k \Phi(i)}{p^{n}}-\sum_{k=0}^{p^{n}-1}\left\langle\frac{m\left(p^{n}-1\right)(\Phi(i)+1)-k \Phi(i)}{p^{n}}\right\rangle \\
& =\sum_{k=0}^{p^{n}-1} \frac{m\left(p^{n}-1\right)(\Phi(i)+1)}{p^{n}}-\sum_{k=0}^{p^{n}-1} \frac{k \Phi(i)}{p^{n}}-\sum_{i=1}^{p^{n}-1}\left\langle\frac{m\left(p^{n}-1\right)(\Phi(i)+1)-k \Phi(i)}{p^{n}}\right\rangle \\
& =m\left(p^{n}-1\right)(\Phi(i)+1)-\frac{\Phi(i)\left(p^{n}-1\right)}{2}-\sum_{k=0}^{p^{n}-1}\left\langle\frac{m\left(p^{n}-1\right)(\Phi(i)+1)-k \Phi(i)}{p^{n}}\right\rangle .
\end{aligned}
$$


As $k$ runs a complete residue system $\bmod p^{n}$ and g.c.d. $(\Phi(i), p)=1$, the same is true for $m\left(p^{n}-1\right)(\Phi(i)+1)-k \Phi(i)$, so

$$
\begin{aligned}
\sum_{k=0}^{p^{n}-1} \nu_{i k}(m) & =m\left(p^{n}-1\right)(\Phi(i)+1)-\frac{\Phi(i)\left(p^{n}-1\right)}{2}-\frac{1}{p^{n}} \sum_{k=0}^{p^{n}-1} k \\
& =m\left(p^{n}-1\right)(\Phi(i)+1)-\frac{\Phi(i)\left(p^{n}-1\right)}{2}-\frac{p^{n}-1}{2} \\
& =m\left(p^{n}-1\right)(\Phi(i)+1)-\frac{\left(p^{n}-1\right)}{2}(\Phi(i)+1) \\
& =(2 m-1)(\Phi(i)+1) \frac{\left(p^{n}-1\right)}{2} .
\end{aligned}
$$

Remark 18. Observe, for one more time that from Eq. (30) we have that

$$
\frac{2}{2 m-1} \sum_{k=0}^{p^{n}-1} \Gamma_{k}(m)=\operatorname{deg} \operatorname{Diff}(F / E) .
$$

Using Eq. (30) and 25, we will then have that

$$
\begin{aligned}
\sum_{k=0}^{p^{n}-1} \Gamma_{k}(m) & =\sum_{k=0}^{p^{n}-1} \sum_{i=1}^{r} \nu_{i k}(m) \\
& =(2 m-1)\left(g_{F}+p^{n}-1\right) .
\end{aligned}
$$

Finally from Eq. 31 we see that

$$
\begin{aligned}
\sum_{k=0}^{p^{n}-1}\left(\Gamma_{k}(m)-2 m+1\right) & =\sum_{k=0}^{p^{n}-1} \Gamma_{k}(m)-2 m p^{n}+p^{n} \\
& =(2 m-1)\left(g_{F}+p^{n}-1\right)-p^{n}(2 m-1) \\
& =(2 m-1)\left(g_{F}-1\right) .
\end{aligned}
$$

Remark 19. If $F / K(x)$ is an extension of degree $p$ (i.e. an Artin Schreier) then Boseck, [3] proved that the set $\Sigma_{1}$ is indeed a basis for $\Omega_{F}(1)$. With exactly the same arguments used here, one can show that the set defined in Proposition 17 is still a basis of $\Omega_{F}(m)$ when $F / K(x)$ is a cyclic Artin Schreier extension of degree $p$.

Remark 20. A basis for the holomorphic $m$-(poly)differentials is closely connected with the computation of the $m$-Weierstrass points of $F$. The case where $m=1$, see Garcia and Boseck ( [8], [7] and [3]), leads to the computation of (classical) Weierstrass points. One can now follow their ideas for the case $m>1$.

3.2. Galois module structure of $\Omega_{F}(\boldsymbol{m})$. In this subsection we will determine the Galois module structure of the space $\Omega_{F}(m)$, of $m$-(poly)differentials using Proposition 17 Following closely the ideas of Calderón, Salvador and Madan ([17], Theorem 1), who considered the case $m=1$, we will generalize their result for $m>1$.

Let $\theta_{0}, \ldots, \theta_{p^{n}-1} \in K$ and for $j=1, \ldots, p^{n}$, let $W_{j}=\left\langle\theta_{0}, \ldots, \theta_{j-1}\right\rangle_{K}$. Let also $G$ acting on these $\theta_{i}$ 's with the action described as follows:

$$
\sigma_{\alpha}\left(\theta_{i}\right)=\sum_{l=0}^{i}\left(\begin{array}{l}
i \\
l
\end{array}\right) \alpha^{i-l} \theta_{l}, \text { for } 0 \leq i \leq k .
$$

In the near future, we will interpret these $\theta_{i}$ 's as sums of specific $m$-holomorphic (poly)differentials (the anxious reader should jump to Eq. (36) ). 
Theorem 21. Let $F / K(x)$ as above. The $K[G]$ module $W_{j}$, for $j=1, \ldots, p^{n}$ is indecomposable, and

$$
\Omega_{F}(m) \simeq \bigoplus_{j=1}^{p^{n}} W_{j}^{d_{j}}
$$

where $d_{p^{n}}$ equals to $\Gamma_{p^{n}-1}(m)-2 m+1$ and is the number of times that the regular representation of $G$ occurs in $\Omega_{F}(m)$, while the indecomposable representation $W_{j}$ occurs $d_{j}=\Gamma_{j-1}(m)-\Gamma_{j}(m)$ times, for all $j=1, \ldots, p^{n}-1$, and for all $m>1$.

Proof. Let $\omega_{k, \nu}^{m}$ be an arbitrary basis element of $\Omega_{F}(m)$ and $\sigma_{\alpha} \in G$ for an $\alpha \in \mathbb{F}_{q}$. Then

$$
\sigma_{\alpha}\left(\omega_{k, \nu}^{m}\right)=x^{\nu}(y+\alpha)^{k}\left[g_{k}(x)\right]^{-1}(d x)^{\otimes m}=\sum_{i=0}^{k}\left(\begin{array}{c}
k \\
i
\end{array}\right) \alpha^{k-i} y^{i} x^{\nu}\left[g_{k}(x)\right]^{-1}(d x)^{\otimes m} .
$$

For $0 \leq i \leq k$, let $h_{i, k}(x)=\prod_{j=1}^{r}\left(x-a_{j}\right)^{\nu_{j i}(m)-\nu_{j k}(m)}$. As $0 \leq i \leq k$, we have that $\nu_{j i}(m) \geq \nu_{j k}(m)$. Thus

$$
K[x] \ni h_{i, k}(x)=\sum_{e=0}^{n(i, k, m)} b_{e}^{(i, k, m)} x^{e},
$$

with $n(i, k, m)=\Gamma_{i}(m)-\Gamma_{k}(m)$ and $b_{n(i, k, m)}^{(i, k, m)}=1$. From Equations (32), (33) and 28) we obtain that

$$
\begin{aligned}
\sigma_{\alpha}\left(\omega_{k, \nu}^{m}\right) & =\sum_{i=0}^{k}\left(\begin{array}{c}
k \\
i
\end{array}\right) \alpha^{k-i} y^{i} x^{\nu}\left[g_{i}(x)\right]^{-1} h_{i, k}(x)(d x)^{\otimes m} \\
& =\sum_{i=0}^{k} \sum_{e=0}^{n(i, k, m)}\left(\begin{array}{c}
k \\
i
\end{array}\right) \alpha^{k-i} y^{i} x^{\nu+e}\left[g_{i}(x)\right]^{-1} b_{e}^{(i, k, m)}(d x)^{\otimes m}
\end{aligned}
$$

Since $0 \leq e \leq \Gamma_{i}(m)-\Gamma_{k}(m)$, we have that $0 \leq \nu+e \leq \Gamma_{i}(m)-2 m$. This means that $y^{i} x^{\nu+e}\left[g_{i}(x)\right]^{-1}(d x)^{\otimes m}$ is a basis element, namely $\omega_{i, \nu+e}^{m}$. Thus, we have that the $G$ action on the basis of $\Omega_{F}(m)$, is given by

$$
\begin{aligned}
\sigma_{\alpha}\left(\omega_{k, \nu}^{m}\right) & =\sum_{i=0}^{k} \sum_{e=0}^{n(i, k, m)}\left(\begin{array}{c}
k \\
i
\end{array}\right) \alpha^{k-i} b_{e}^{(i, k, m)} \omega_{i, \nu+e}^{m} \\
& =\sum_{i=0}^{k}\left(\begin{array}{c}
k \\
i
\end{array}\right) \alpha^{k-i}\left\{\sum_{e=0}^{n(i, k, m)} b_{e}^{(i, k, m)} \omega_{i, \nu+e}^{m}\right\} .
\end{aligned}
$$

Observe that the coefficient of $\omega_{k, \nu}^{m}$ in the right side of Eq. (34) is 1 .

Let $M_{k, \nu}^{m}$, be the $K[G]$-module, generated by

$$
\left\{\omega_{i, \nu+e}^{m} \mid 0 \leq i \leq k, 0 \leq e \leq n(i, k, m)\right\} .
$$

The following cases play an important role in the decomposition of $M_{k, \nu}^{m}$, which will follow:

- The condition $i=k$, implies $e=0$ and the differentials of $M_{k, \nu}^{m}$ which satisfy that condition, are of the form $\left\{\omega_{k, \nu}^{m}\right\}$, while

- The conditions $e=0$ and $i \neq k$ are satisfied by the differentials of $M_{k, \nu}^{m}$, of the form $\left\{\omega_{i, \nu}^{m} \mid 0 \leq i \supsetneqq k\right\}$.

With these in mind, we have that

$$
M_{k, \nu}^{m}=N_{k, \nu}^{m} \oplus U_{k, \nu}^{m},
$$


as $K[G]$-modules, where $N_{k, \nu}^{m}$ and $U_{k, \nu}^{m}$ are generated respectively, by the sets

$$
\left\{\omega_{i, \nu+e}^{m} \mid 0 \leq i \supsetneqq k, 0 \supsetneqq e \leq n(i, k, m)\right\} \text { and }\left\{\theta_{0}, \ldots, \theta_{k}\right\} \text {, }
$$

where

$$
\begin{aligned}
\theta_{i} & :=\theta_{i}^{(k, \nu, m)} \\
(36) & =\sum_{e=0}^{n(i, k, m)} b_{e}^{(i, k, m)} \omega_{i, \nu+e}^{m}= \begin{cases}b_{0}^{(i, k, m)} \omega_{i, \nu}^{m}+\sum_{e=1}^{n(i, k, m)} b_{e}^{(i, k, m)} \omega_{i, \nu+e}^{m}, & \text { if } i \neq k, \\
\omega_{k, \nu}^{m}, & \text { if } i=k .\end{cases}
\end{aligned}
$$

Notice that the decomposition in Eq. (35) is a decomposition of $K$-vector spaces since we can select our model so that $b_{0} \neq 0$, see Remark 22.

The $K[G]$ action on $N_{k, \nu}^{m}$ 's is given by

$$
\sigma_{\alpha}\left(\omega_{i, \nu+e}^{m}\right)=\sum_{\delta=0}^{i} \sum_{\theta=0}^{n(\delta, i, m)}\left(\begin{array}{l}
i \\
\delta
\end{array}\right) \alpha^{i-\delta} b_{\theta}^{(\delta, i, m)} \omega_{\delta, \nu+e+\theta}^{m} .
$$

Observing that

$$
\begin{aligned}
0 \supsetneqq e+\theta & \leq n(i, k, m)+n(\delta, i, m) \\
& =\Gamma_{i}(m)-\Gamma_{k}(m)+\Gamma_{\delta}(m)-\Gamma_{i}(m) \\
& =n(\delta, k, m),
\end{aligned}
$$

we see that the action is well defined, i.e. $\omega_{\delta, \nu+e+\theta}^{m} \in N_{k, \nu}^{m}$. In a similar way, using Eq. (34) for the $\theta_{i}$ 's (compare also to [17, p. 153, Eq. (7)]), we see that the $K[G]$ action on $U_{k, \nu}^{m}$ is given by

$$
\sigma_{\alpha}\left(\theta_{i}\right)=\sum_{l=0}^{i}\left(\begin{array}{l}
i \\
l
\end{array}\right) \alpha^{i-l} \theta_{l}, \text { for } 0 \leq i \leq k .
$$

Thus the spaces $N_{k, \nu}^{m}$ and $U_{k, \nu}^{m}$ are indeed $K[G]$ modules and Eq. [35], is actually a $K[G]$ module decomposition of $M_{k, \nu}^{m}$.

Remark 22. In Eq. (36), where we define the thetas, we can assume that $b_{0}^{(i, k, m)} \neq 0$, in order to have the desired terms $\omega_{i, \nu}^{m}$. Indeed $b_{0}^{(i, k, m)}$ is just the constant term of the polynomial $h_{i, k}(x)=\prod_{j=1}^{r}\left(x-a_{j}\right)^{\nu_{j i}(m)-\nu_{j k}(m)}$, which is zero when any of its roots $a_{j}$ equals to zero. Thus we may assume, after a birational transformation (i.e. an appropriate translation), that $a_{j} \neq 0$ in Eq. (23), for all $1 \leq j \leq r$.

We now present a counting argument:

Let $0 \leq k_{0} \leq p^{n}-1$ be maximal such that $\Gamma_{k_{0}}(m)-2 m \geq 0$ and set $0 \leq \Gamma_{k_{0}}(m)-2 m=$ $\nu_{0}$. Observe that with the above hypothesis $k_{0}=p^{n}-1$. If we were in the case $m=1$ an admissible value for $k_{0}$ would be $p^{n}-2$.

Recall that $\Omega_{L}(m)$ is generated by

$$
\left\{\omega_{k, \nu}^{m} \mid 0 \leq k \leq k_{0}, 0 \leq \nu \leq \Gamma_{k}(m)-2 m\right\} .
$$

Claim:

$$
\Omega_{L}(m)=N_{k_{0}, \nu_{0}}^{m} \bigoplus_{j=0}^{\nu_{0}} U_{k_{0}, j}^{m}
$$

as $K[G]$ modules, with $N_{k_{0}, \nu_{0}}$ generated by

$$
\left\{\omega_{k, \nu}^{m} \mid 0 \leq k \supsetneqq k_{0}, 0 \leq \nu_{0} \supsetneqq \nu \leq \Gamma_{k}(m)-2 m\right\}
$$

and for every $0 \leq j \leq \nu_{0}$, each $U_{k_{0}, j}^{m}$ is generated by

$$
\left\{\theta_{i}^{\left(k_{0}, j, m\right)} \mid 0 \leq i \leq k_{0}\right\},
$$


where $\theta_{i}^{\left(k_{0}, j, m\right)}$,s are given by Eq. (36) .

Proof of the Claim:

Note that $\operatorname{dim}_{K} U_{k_{0}, j}^{m}=k_{0}+1$, for all $0 \leq j \leq \nu_{0}$.

Remember, that $\theta_{i}^{\left(k_{0}, j, m\right)}$ equals to

$$
\begin{aligned}
\theta_{i}^{\left(k_{0}, j, m\right)} & =\sum_{e=0}^{n\left(i, k_{0}, m\right)} b_{e}^{\left(i, k_{0}, m\right)} \omega_{i, j+e}^{m} \\
& = \begin{cases}b_{0}^{\left(i, k_{0}, m\right)} \omega_{i, j}^{m}+\sum_{e=1}^{n\left(i, k_{0}, m\right)} b_{e}^{\left(i, k_{0}, m\right)} \omega_{i, j+e}^{m}, & \text { if } i \neq k_{0}, \\
\omega_{k_{0}, j}^{m}, & \text { if } i=k_{0},\end{cases}
\end{aligned}
$$

We need the followings Propositions in order to prove the claim:

Proposition 23. $U_{k_{0}, j}^{m} \cap N_{k_{0}, \nu_{0}}^{m}=\{0\}$, for all $0 \leq j \leq \nu_{0}$.

Proof. According to Eq. (41), every $\theta_{i}^{\left(k_{0}, j, m\right)}$, would contain as a summand an $b_{0}^{\left(i, k_{0}, m\right)} \omega_{i, j}^{m}$, with $0 \leq j \leq \nu_{0}$, but from the definition of $N_{k_{0}, \nu_{0}}^{m}$, Eq. (40), these elements are not in $N_{k_{0}, \nu_{0}}^{m}$. These elements $b_{0}^{\left(i, k_{0}, m\right)} \omega_{i, j}^{m}$ cannot be canceled out by linear combinations of elements in $U_{k_{0}, j}^{m}$. Therefore no linear combination of $\theta_{i}^{\left(k_{0}, j, m\right)}$ can be in $N_{k_{0}, \nu_{0}}^{m}$.

Proposition 24. $U_{k_{0}, j}^{m} \cap U_{k_{0}, j^{\prime}}^{m}=\{0\}$, for every $j \neq j^{\prime}$, with $0 \leq j \supsetneqq j^{\prime} \leq \nu_{0}$.

Proof. Fix a $j$ and let $j^{\prime} \neq j$. We may also assume that $j \supsetneqq j^{\prime}$. We consider a linear combination of elements $\theta_{i}^{\left(k_{0}, j^{\prime}, m\right)}$ in $U_{k_{0}, j^{\prime}}^{m}$.

Under the assumption $j \supsetneqq j^{\prime}$, Eq. (41) tells us that $b_{0}^{\left(i, k_{0}, m\right)} \omega_{i, j}^{m}$, a summand of a linear combination of $\theta_{i}^{\left(k_{0}, j, m\right)}$, is not a summand of a linear combination of the elements $\theta_{i}^{\left(k_{0}, j^{\prime}, m\right)}$, i.e $\omega_{i, j}^{m} \notin\left\langle\theta_{i}^{\left(k_{0}, j^{\prime}, m\right)}\right\rangle_{0 \leq i \leq k_{0}}$.

Finally, observe that the $M_{k_{0}, \nu_{0}}$ is a $\Omega_{L}(m)$ submodule of co-dimension $\left(k_{0}+1\right) \nu_{0}$ and, from Eq. 35], the same is true for $N_{k_{0}, \nu_{0}}^{m} \oplus U_{k_{0}, \nu_{0}}^{m}$. The claim is then proved using Propositions 23 and 24, if we notice that $\operatorname{dim}_{K} \bigoplus_{j=0}^{\nu_{0}-1} U_{k_{0}, j}^{m}=\nu_{0}\left(k_{0}+1\right)$.

Observe also, using the Eq. (38), that for every $j$ the $U_{k_{0}, j}^{m}$, s that appear in Eq. (39), are $K[G]$ isomorphic. For example, using again Eq. [36, we can construct an isomorphism $f: U_{k_{0}, 0}^{m} \longrightarrow U_{k_{0}, 1}^{m}$ as follows; $f$ maps $\omega_{i, \nu} \mapsto \omega_{i, \nu+1}$, with $0 \leq \nu \leq \nu_{0}$ and $0 \leq i \leq k_{0}$. So we can drop the $j$ subscript on the notation of $U_{k_{0}, j}^{m}$ and think of the $\bigoplus_{j=0}^{\nu_{0}} U_{k_{0}, j}^{m}$ as $\nu_{0}+1$ copies of $U_{k_{0}}^{m}$. Then, rewriting Eq. 39) we have that

$$
\Omega_{L}(m)=N_{k_{0}, \nu_{0}}^{m} \oplus\left[U_{k_{0}}^{m}\right]^{\Gamma_{k_{0}}-2 m+1} .
$$

That finishes the zeroth step of the proof of the Theorem. Then we proceed to the first step. We take $N_{k_{0}, \nu_{0}}^{m}$, in place of $\Omega_{F}(m)$ : Let $0 \leq k_{1} \supsetneqq k_{0}=p^{n}-1$ be maximal such that $\Gamma_{k_{1}}(m)-2 m \geq 0$ and set $0 \leq \Gamma_{k_{1}}(m)-2 m=\nu_{1}$. Then, repeating the claim in the previous step we, can see that

$$
N_{k_{0}, \nu_{0}}^{m}=N_{k_{1}, \nu_{1}}^{m} \bigoplus_{j=0}^{\nu_{1}} U_{k_{1}, j}^{m},
$$

where $N_{k_{1}, \nu_{1}}^{m}$ is generated by

$$
\left\{\omega_{k, \nu}^{m} \mid 0 \leq k \supsetneqq k_{1}, 0 \leq \nu_{0} \supsetneqq \nu_{1} \supsetneqq \nu \leq \Gamma_{k}(m)-2 m\right\}
$$

and for every $0 \leq j \leq \nu_{1}$, each $U_{k_{1}, j}^{m}$ is generated by

$$
\left\{\theta_{i}^{\left(k_{1}, j, m\right)} \mid 0 \leq i \leq k_{1}\right\}
$$

with $\theta_{i}^{\left(k_{1}, j, m\right)}$, s are given always by Eq. (36). Note that for $0 \leq j \leq \nu_{1}, \operatorname{dim}_{K} U_{k_{1}, j}^{m}=$ $k_{1}+1$ and all the $U_{k_{1}, j}^{m}$ 's that appearing in Eq. (43) are $K[G]$ isomorphic. There are 
exactly $\nu_{1}-\nu_{0}$ such modules, with $0 \supsetneqq \nu_{1}-\nu_{0}=\Gamma_{k_{1}}(m)-\Gamma_{k_{0}}(m)$. We can rewrite Eq. (43), dropping the $j$ subscript, and thinking $\bigoplus_{j=0}^{\nu_{1}} U_{k_{1}, j}^{m}$ as $\Gamma_{k_{1}}(m)-\Gamma_{k_{0}}(m)$ copies of $U_{k_{1}}$ :

$$
N_{k_{0}, \nu_{0}}^{m}=N_{k_{1}, \nu_{1}}^{m} \oplus\left[U_{k_{1}}^{m}\right]^{\Gamma_{k_{1}}(m)-\Gamma_{k_{0}}(m)} .
$$

Now we apply the above argument recursively to $N_{k_{\zeta}, \nu_{\zeta}}^{m}$, for $0 \leq \zeta \leq p^{n}-1$, continuing the above decomposition and replacing always the $N_{k_{\zeta}, \nu_{\zeta}}^{m}$ with the $N_{k_{\zeta-1}, \nu_{\zeta-1}}^{m}$.

From Eq. 42), (44) and the repeated procedure, we are now able to express $\Omega_{F}(m)$ as a direct sum of $U_{k_{\zeta}, \nu_{\zeta}}^{m}$ 's. Collecting these $K[G]$ modules of the same dimension we have

$$
\Omega_{F}(m) \simeq \oplus_{\zeta=0}^{p^{n}-1}\left[U_{k_{\zeta}}^{m}\right]^{\Lambda_{k_{\zeta}}}
$$

with $\Lambda_{k_{0}}=\Gamma_{p^{n}-1}(m)-2 m+1$ and $\Lambda_{k_{\zeta}}=\Gamma_{k_{\zeta}}(m)-\Gamma_{k_{\zeta-1}}(m)$, for all the steps: $1 \leq \zeta \leq p^{n}-1$. From Eq. (38) all $U_{k_{\zeta}}^{m}$ with the same dimension, say $j$, are $K[G]$ isomorphic, thus are isomorphic with $U_{j-1}^{m}$. We re-index in order to be consistent with the dimension, letting $j-1=k_{\zeta}$ (observe that with this setting $k_{\zeta}+1=k_{\zeta-1}$ ), we obtain

$$
\begin{aligned}
& \Omega_{F}(m) \simeq \oplus_{j=1}^{p^{n}}\left[U_{j-1}^{m}\right]^{\Lambda_{j-1}}, \text { or } \\
& \Omega_{F}(m) \simeq \oplus_{j=1}^{p^{n}} T_{j} .
\end{aligned}
$$

The module $T_{p^{n}}$ is a direct sum of $\Gamma_{p^{n}-1}(m)-2 m+1$ modules of dimension $p^{n}$ and $T_{j}$ is a direct sum of $\Gamma_{j-1}(m)-\Gamma_{j}(m)$ modules of dimension $j$, with $1 \leq j \leq p^{n}-1$.

We will now prove that the modules $U_{j-1}^{m}$ are indeed indecomposable.

Let $\Omega_{F}(m) \simeq \oplus_{i=1}^{\eta} M_{i}$, be a decomposition in indecomposable $K[G]$-modules of the space of holomorphic $m$-(poly)differentials. Then

$$
\eta \geq \sum_{j=1}^{p^{n}-1}\left(\Gamma_{j-1}(m)-\Gamma_{j}(m)\right)+\Gamma_{p^{n}-1}(m)-2 m+1=\Gamma_{0}(m)-2 m+1 .
$$

Since $G$ is a $p$-group, we know that $\tau$, the one-dimensional trivial representation, is the only irreducible representation of $G$ (see [24, p.187, Proposition 1.1]). Then, if $M_{i}^{G}$ denote the $K[G]$-submodule of fixed points of $M_{i}, M_{i}^{G}$ would contain $\tau$ as a subrepresentation, so $\operatorname{dim}_{K} M_{i}^{G} \geq 1$. Thus

$$
\eta \leq \operatorname{dim}_{K} \Omega_{F}^{G}(m) .
$$

It is well known (see for example [6, p. 271, V.2.2], or [19, p.83, Theorem III.4.6]), that differentials of $K(x)$ can be lifted to $G$-invariant differentials of $F$ via the Cotrace map. So $\Omega_{F}^{G}(m)=\left\{\kappa(d x)^{\otimes m} \mid \kappa \in K(x)\right.$ with $\left.\operatorname{div}_{F}\left(\kappa(d x)^{\otimes m}\right) \geq 0\right\}$ and we have

$$
\begin{aligned}
\operatorname{div}_{F}\left(\operatorname{Cotr}_{F / K(x)}\left(\kappa(d x)^{\otimes m}\right)\right) & :=\operatorname{div}_{F}\left(\kappa(d x)^{\otimes m}\right) \\
& =\operatorname{Con}_{F / K(x)}\left(\operatorname{div}_{K}\left(\kappa(d x)^{\otimes m}\right)\right)+m \operatorname{Diff}(F / K(x)) .
\end{aligned}
$$

Evaluating Eq. (48), for the places $P_{i} \in \mathbb{P}_{F}$ we have for all $1 \leq i \leq r$ and for all $\kappa(d x)^{\otimes m} \in \Omega_{F}^{G}(m)$, that

$$
v_{P_{i}}\left(\operatorname{div}_{F}\left(\kappa(d x)^{\otimes m}\right)\right)=p^{n} v_{p_{i}}(\kappa)+m(\Phi(i)+1)\left(p^{n}-1\right) \geq 0,
$$

So

$$
\begin{aligned}
& v_{p_{i}}(\kappa) \geq-\frac{m(\Phi(i)+1)\left(p^{n}-1\right)}{p^{n}}, \text { or using Eq. (27) } \\
& v_{p_{i}}(\kappa) \geq-\nu_{i 0}(m), \text { for all } 1 \leq i \leq r .
\end{aligned}
$$

For $Q \in \mathbb{P}_{K(x)}$, with $Q \neq p_{i}, p_{\infty}$, taking $\kappa(d x)^{\otimes m} \in \Omega_{F}^{G}(m)$ and using Eq. (48) and (24), we have that

$$
v_{Q}(\kappa) \geq 0
$$


while for the infinite place of $K(x)$, the same hypothesis and Equations yield

$$
v_{p_{\infty}}(\kappa) \geq 2 m \text {. }
$$

Gathering Eq. (49), (50) and (51), we can write $\Omega_{F}^{G}(m)$ in an alternative form, namely

$$
\Omega_{F}^{G}(m)=\left\{\frac{c(x)}{\prod_{i=1}^{r}\left(x-a_{i}\right)^{\nu_{i 0}(m)}} \mid c(x) \in K[x], \operatorname{deg} c(x) \leq \sum_{i=1}^{r} \nu_{i 0}(m)-2 m\right\} .
$$

Therefore $\operatorname{dim}_{K} \Omega_{F}^{G}(m)=\Gamma_{0}(m)-2 m+1$. Using this fact, together with Eq. 460 and (47) we have that $\eta=\Gamma_{0}(m)-2 m+1$. This shows that the $K[G]$-modules appearing to each $T_{j}$ in the decomposition of $\Omega_{F}(m)$ at Eq. (45), are all indecomposable for every $1 \leq j \leq p^{n}$.

The theorem follows by letting $W_{j}=U_{j-1}^{m}$ and $d_{j}=\Lambda_{j-1}$.

Remark 25. Another way to see the indecomposability of the $W_{j}$ 's, for $j \neq 0$, is to notice that if $W_{j}$, were decomposable, say $W_{j}=M_{1} \oplus M_{2}$, then each $M_{i}$ would contain a copy of $U_{0, \nu}^{m}$, and $W_{j}$ would contain $U_{0, \nu}^{m} \oplus U_{0, \nu}^{m}$ as a subrepresentation. But since $\operatorname{dim}_{K} W_{j}^{G} \leq 1$, $\left(\operatorname{dim}_{K} W_{j}^{G} \leq \operatorname{dim}_{K} K[G]^{G}=1\right)$, that is a contradiction.

\section{A CONJECTURE CONCERNING ABELIAN GROUPS OF ORDER $p^{n}$.}

We strongly believe that for an arbitrary Galois $p$-extension, $F / E$, with abelian Galois group, one can calculate explicitly the $K[G]$-module structure of the space of holomorphic $m$-(poly)differentials:

Conjecture 26. Let $G$, be a p-group of automorphisms of $F$. Set $E=F^{G}$ and let $g_{E}$ be the genus of $E$ and $g_{F} \geq 2$, the genus of $F$. Let $m$ be a natural number with $m>1, \delta_{i}$ the different exponent of the extension and $e_{i}$ the ramification indices of the ramified primes of $E$ in $F$. The regular representation of $G$ occurs $\Gamma_{p^{n}-1}(m)+\left(g_{E}-1\right)(2 m-1)$ times in the representation of $G$ on $\Omega_{F}(m)$. For $k=1, \ldots,[F: E]-1$, the indecomposable representation of degree $k$ occurs $\Gamma_{k-1}(m)-\Gamma_{k}(m)$ times. Where $\Gamma_{k}(m)=\sum_{i} \nu_{i k}(m)$, are the Boseck invariants, with $i$ running over the ramified primes of $E$ in $F$ and the quantities $\nu_{i k}(m)$, are defined to be

$$
\left\lfloor\frac{m \delta_{i}+\{\text { evaluation of the } k t h \text { E- basis element of } F \text { by a normalized valuation of } F\}}{p^{e_{i}}}\right\rfloor \text {, }
$$

where the basis element is evaluated by a (normalized) valuation determined by a place of $F$ above a ramified place of $E$ and $\lfloor\cdot\rfloor$ denotes the integer part.

For the case $m=1$ the above Conjecture has been proved in some cases, since has the form:

\section{Conjecture 27.}

(i) Wild ramification: If $G$ is a p-group, $m=1$ and there is a place in $\mathbb{P}_{E}$ that is totally ramified in $F$, with $F=E^{G}$, then the regular representation of $G$ occurs $d_{p^{n}}:=g_{E}$ times in the representation of $G$ on $\Omega_{F}(1)$. For $k=1, \ldots, p^{n}-1$, the indecomposable representation of degree $k$ occurs $d_{k}:=\Gamma_{k-1}(1)-\Gamma_{k}(1)+\beta$ times, where $\beta$ equals to -1 , if $k=p^{n}-1$ and equals to zero otherwise. The Boseck invariants are defined as before, by letting $m=1$.

(ii) Tame ramification: If $m=1$ and $F / E$ is ramified of degree $n$, with g.c.d. $(n, p)=$ 1 , then for $k=0, \ldots, n-1$, we have $n$ distinct irreducible representations of degree 1 . The $k$ th representation occurs $d_{n-k}:=\Gamma_{n-k}(1)-1+g_{E}+\beta$ times in the representation of $G$ in $\Omega_{F}(1)$, where $\beta$ equals to 1 , if $k=0$ and zero 
otherwise. The $\Gamma_{k}(1)=\sum_{i} \nu_{i k}(1)$, are the Boseck invariants, with $i$ running over the ramified primes of $E$ in $F$ and the quantities $\nu_{i k}(m)$, are defined to be $\left\langle\frac{\{\text { evaluation of the } k \text { th } E \text { - basis element of } F \text { by a normalized valuation of } F\}}{e_{i}}\right\rangle$,

where $e_{i}$ is the corresponding ramification index and $\langle\cdot\rangle$ denote the fractional part of .

The first case is proved when $G$ is cyclic or an elementary abelian of order $p^{n}$. The second case is Hurwitz's Theorem (see Theorem 15. Note that in all cases, Boseck invariants $\Gamma_{k}(m)$, defined to be the quantities that come out from Boseck's bases.

Let $\mathscr{C}$ denote the Cartier operator (see for example [14, p. 349]), then from the theory of $\frac{1}{p}$-linear maps, it is well known that $\Omega_{F}(1)$ decomposes as

$$
\Omega_{F}(1)=\Omega_{F}^{s}(1) \bigoplus \Omega_{F}^{n}(1),
$$

where $\Omega_{F}^{s}(1)$ denotes the semisimple part of $\Omega_{F}(1)$, that is the $K$ vector space spanned by the set $\left\{\omega \in \Omega_{F}(1) \mid \mathscr{C} \omega=\omega\right\}$, and $\Omega_{F}^{n}(1)$ denotes the nilpotent part, the $K$ vector space spanned by $\left\{\omega \in \Omega_{F}(1) \mid \mathscr{C}^{i} \omega=0\right.$, for some $\left.i \geq 1\right\}$. Now if the Conjecture 27 (i) is proved then, coupled with the main result of [14], will give explicitly the structure of the nilpotent part of $\Omega_{F}(1)$, a problem that is open, as far as we know. Notice finally that we can calculate the nilpotent part of $\Omega_{F}(1)$ in both the elementary abelian and the cyclic case, i.e. for the cases that this Conjecture has already been proved, combining the results of [23], [17] and [14].

The reason we believe that this conjecture is true is that we are able to prove it for the two extreme cases of abelian groups of order $p^{n}$, namely elementary abelian groups $\mathbb{Z} / p \mathbb{Z} \times \cdots \times \mathbb{Z} / p \mathbb{Z}$ and cyclic groups $\mathbb{Z} / p^{n} \mathbb{Z}$. Since $G$ is abelian we can decompose it to a direct sum of cyclic $p$-groups. Thus a function field having $G$ as its Galois group can result as the compositum of (some) cyclic function fields found in subsection 2.1 with elementary abelian function fields from section 3 . A difficult that now arises for such extensions is that we do not know how Boseck invariants, and hence bases (even in the $m=1$ case) behave under such compositums.

\section{AN APPLICATION TO LOCAL DEFORMATION FUNCTORS}

Let $G$ be a $p$-group. It was observed in [11] that the tangent space of the global deformation functor $H^{1}\left(G, \mathscr{T}_{X}\right)$ can be computed in terms of covariants of 2-holomorphic differentials by

$$
H^{1}\left(G, \mathscr{T}_{X}\right)=\Omega_{X}^{\otimes 2} \otimes_{K[G]} K
$$

In this section we will use the results we obtained so far in order to express the dimension of the above spaces in terms of the Boseck invariants. We will use the global deformation functor approach in order to study the tangent space $H^{1}\left(G, \mathscr{T}_{K[[t]]}\right)$ of the local deformation functor in the sense of J.Bertin and A. Mézard [1]. This can be done by considering Katz-Gabber covers [10] of the projective line, i.e. Galois cover $\pi: X \rightarrow \mathbb{P}^{1}$ with only one full ramification point and Galois group $G$. For first order infinitesimal deformations of the curve $X$ with the automorphism group $G$, there is a splitting of the tangent space $H^{1}\left(G, \mathscr{T}_{X}\right)$ :

$$
H^{1}\left(G, \mathscr{T}_{X}\right)=H^{1}\left(X / G, \pi_{*}^{G}\left(\mathscr{T}_{X}\right)\right) \oplus H^{1}\left(G, \mathscr{T}_{K[[t]]}\right) .
$$

For the dimension of the space $H^{1}\left(X / G, \pi_{*}^{G}\left(\mathscr{T}_{X}\right)\right)$ we have an explicit formula, namely

$$
\operatorname{dim}_{K} H^{1}\left(X / G, \pi_{*}^{G}\left(\mathscr{T}_{X}\right)\right)=3 g_{X / G}-3+\left\lceil\frac{\delta}{p^{n}}\right\rceil,
$$

where $\delta$ is the local contribution to the different at the unique ramification point [13, Eq. (38)]. 
Case 1. The group $G$ is cyclic. In this case each of the indecomposable components of Theorem 6 has an one dimensional covariant subspace thus

$$
\begin{aligned}
\operatorname{dim}_{K} \Omega_{X}^{\otimes 2} \otimes_{K[G]} K & =\sum_{\nu=1}^{p^{n}} d_{\nu} \\
& =3\left(g_{X / G}-1\right)+\Gamma_{0}(2)=-3+\left\lfloor\frac{2 \delta}{p^{n}}\right\rfloor .
\end{aligned}
$$

Using Eq. (53), 54, (55) we arrive at

$$
\operatorname{dim}_{K} H^{1}\left(G, \mathscr{T}_{K[[t]]}\right)=\left\lfloor\frac{2 \delta}{p^{n}}\right\rfloor-\left\lceil\frac{\delta}{p^{n}}\right\rceil,
$$

which coincides with the computation of [1, Prop. 4.1.1].

Case 2. The group $G$ is elementary abelian. In this case we will use Theorem 21 in order to arrive to

$$
\operatorname{dim}_{K} H^{1}\left(G, \mathscr{T}_{K[[t]]}\right)=\sum_{j=1}^{p^{n}} d_{j} \cdot \operatorname{dim}_{K}\left(W_{j} \otimes_{K[G]} K\right)+3-\left\lceil\frac{\delta}{p^{n}}\right\rceil .
$$

Proposition 28. For the dimension $W_{j} \otimes_{K[G]} K$ we compute

$$
\operatorname{dim}_{K} W_{j} \otimes_{K[G]} K= \begin{cases}1 & \text { if } 1 \leq j \leq p \\ 2 & \text { if } p+1 \leq j \leq p^{n} \text { and } j \neq 0 \bmod p \\ 1 & \text { if } j=0 \bmod p\end{cases}
$$

Proof. We identify an elementary abelian group of order $p^{n}$ with the additive group of the field $\mathbb{F}_{p^{n}}$. The field $\mathbb{F}_{p^{n}}$ is an $\mathbb{F}_{p}$ vector space with basis $1, e, e^{2}, \ldots, e^{n-1}$ for some element $e$. Every element $a \in \mathbb{F}_{p^{n}}$ gives rise to an automorphism $\sigma_{a}$. We will denote by $\bar{W}_{j}=W_{j} \otimes_{K[G]} K$. The modules $\bar{W}_{j}$ are given by $W_{j} /\left(\sigma_{a}(w)-w\right)$, where $a$ runs over $\mathbb{F}_{p^{n}}$ and $w$ runs over $W_{j}$.

The module $W_{1}=\left\langle\theta_{0}\right\rangle$ and is already $G$-invariant. Observe that $W_{2}=\left\langle\theta_{0}, \theta_{1}\right\rangle$ and the action is given by $\sigma_{a}\left(\theta_{0}\right)=\theta_{0}, \sigma_{a}\left(\theta_{1}\right)=\theta_{1}+a \theta_{0}$. Therefore we have only one relation in the module of covariants $\bar{W}_{2}$ namely $\sigma_{a}\left(\theta_{1}\right)-\theta_{1}=a \theta_{0}$ which implies that the image $\bar{\theta}_{0}$ in $\bar{W}_{2}$ is zero.

The module $W_{3}$ is generated by $\theta_{0}, \theta_{1}, \theta_{2}$ and the relation $\bar{\theta}_{0}=0$ is inherited in $\bar{W}_{3}$. We also have the relation $\sigma_{a}\left(\theta_{2}\right)=\theta_{2}+a \theta_{1}+a^{2} \theta_{0}$, which implies that $\bar{\theta}_{1}=0$ in $\bar{W}_{3}$.

We proceed by induction. For the inductive step we assume that for $j+1 \leq p$ we have the relation $\bar{\theta}_{0}, \ldots, \bar{\theta}_{j-2}=0$ in $\bar{W}_{j}$. Then $\sigma_{a}\left(\theta_{j}\right)=\theta_{j}+a \theta_{j-1}+L$ where $L$ is an $\mathbb{F}_{p^{n}}$ linear combination of $\theta_{\nu}$ with $\nu \leq j-2$ that have zero image in the module of covariants. Thus $\bar{\theta}_{j-1}=0$ in $\bar{W}_{j+1}$ and $\bar{W}_{j+1}=\left\langle\bar{\theta}_{j}\right\rangle$.

For the module $W_{p+1}$ the situation changes: We have $\sigma_{a}\left(\theta_{p}\right)=\theta_{p}+a^{p} \theta_{0}$, which does not give any new relation. Therefore $\bar{W}_{p+1}$ is two dimensional generated by $\bar{\theta}_{p-1}, \bar{\theta}_{p}$.

We proceed by induction. For the modules $\bar{W}_{p+\nu+1}, 1 \leq \nu<p-1$ the inductive hypothesis is that $\bar{W}_{p+\nu+1}=\left\langle\bar{\theta}_{p-1}, \bar{\theta}_{p+\nu}\right\rangle$. We compute that for $1<\nu \leq p-1$

$$
\sigma_{a}\left(\theta_{p+\nu}\right)=\sum_{\mu=1}^{\nu}\left(\begin{array}{l}
p+\nu \\
p+\mu
\end{array}\right) a^{\nu-\mu} \theta_{p+\mu}+\left(\begin{array}{c}
p+\nu \\
p-1
\end{array}\right) a^{\nu+1} \theta_{p-1} .
$$

Notice that according to [5, prop. 15.21] $\left(\begin{array}{l}p+\nu \\ p-1\end{array}\right)=0$ unless $\nu=p-1$. Therefore for $\nu<p-2$ and after some computations, we arrive at

$$
\sigma_{a}\left(\theta_{p+\nu+1}\right)=\theta_{p+\nu+1}+\nu a \theta_{p+\nu}
$$

and this implies that $\bar{\theta}_{p+\nu}=0$ in $\bar{W}_{p+\nu+2}$, thus $\bar{W}_{p+\nu+2}=\left\langle\bar{\theta}_{p-1}, \bar{\theta}_{p+\nu+1}\right\rangle$.

Since $\bar{W}_{2 p-1}=\left\langle\bar{\theta}_{p-1}, \bar{\theta}_{2 p-2}\right\rangle$, we can now compute from Eq. 57 for $\nu=p-1$,

$$
\sigma\left(\theta_{2 p-1}\right)=\theta_{2 p-1}-a \theta_{2 p-2}+a^{p} \theta_{p-1} \text {. }
$$


Therefore in $\bar{W}_{2 p}$ we have the relations $a \bar{\theta}_{2 p-2}=a^{p} \bar{\theta}_{p-1}$ for $a \in \mathbb{F}_{p^{n}}$. Taking $a=1$ we obtain $\bar{\theta}_{2 p-2}=\bar{\theta}_{p-1}$ and then by taking $a=e$ we have $\bar{\theta}_{2 p-2}=e^{p-1} \bar{\theta}_{2 p-2}$, therefore $\bar{\theta}_{p-1}=\bar{\theta}_{2 p-2}=0$. Thus $\bar{W}_{2 p}=\left\langle\bar{\theta}_{2 p-1}\right\rangle$.

We now continue to $\bar{W}_{2 p+1}$ by computing that

$$
\sigma_{a}\left(\theta_{2 p}\right)=\theta_{2 p}+2 a^{p} \theta_{p}+a^{p^{2}} \theta_{0},
$$

thus $\bar{W}_{2 p+1}=\left\langle\bar{\theta}_{2 p-1}, \bar{\theta}_{2 p}\right\rangle$ is of dimension 2 .

If $n \geq 2$ we proceed the same way: The modules $\bar{W}_{2 p+\nu}, 1 \leq \nu \leq p-1$ are 2-dimensional and $\bar{W}_{3 p}$ is one dimensional. A final inductive argument shows that the $\bar{W}_{\lambda p+\nu}$, for $\lambda \leq p^{n-1}$ have the desired dimensions.

We now can give a closed formula for the sum given in eq. (56). By the construction of the Katz-Gabber cover there is only one ramified point and we are interested for 2holomorphic differentials $(m=2)$ so we set

We compute:

$$
\Gamma_{j}(2)=\Gamma_{j}:=\left\lfloor\frac{2\left(p^{n}-1\right)(\Phi+1)+j \Phi}{p^{n}}\right\rfloor .
$$

$$
\begin{gathered}
\sum_{j=1}^{p^{n}} d_{j} \cdot \operatorname{dim}_{K}\left(W_{j} \otimes_{K[G]} K\right)= \\
=\sum_{j=1}^{p-1} d_{j} \cdot \operatorname{dim}_{K}\left(W_{j} \otimes_{K[G]} K\right)+\sum_{j=p}^{p^{n}} d_{j} \cdot \operatorname{dim}_{K}\left(W_{j} \otimes_{K[G]} K\right)= \\
=\Gamma_{0}-\Gamma_{p-1}+2\left(\Gamma_{p-1}-3\right)-\sum_{\nu=0}^{p^{n}-1}\left(\Gamma_{\nu-1}-\Gamma_{\nu}\right)= \\
=\Gamma_{0}+\Gamma_{p-1}-6-\sum_{\substack{\nu=0 \\
p \mid \nu}}^{p^{n}-1}\left(\Gamma_{\nu-1}-\Gamma_{\nu}\right) .
\end{gathered}
$$

This formula should give the same results with the formula given in [13]. Giving a direct proof that the two formulas coincide is a complicated task to do. However using the Magma algebra system we checked that Eq. (58) coincides with the formula given in [13], for all choices of $G, p$ that we tried. The magma [4] program used to compute them is available at http://myria.math. aegean.gr/ kontogar/sk/.

Remark: If the conjectures given in section 4 are proved then we have a method to compute the tangent space for the deformation space of curves with automorphism in the case of abelian groups.

Acknowledgment. The author would like to thank A. Kontogeorgis for all his valuable comments while reading earlier versions of this paper, for the enlightening conversations we had, and for being a wonderful companion during this first journey to mathematical research. In section 5, the work needed in order to confirm our results is completely due to him.

\section{REFERENCES}

1. José Bertin and Ariane Mézard, Déformations formelles des revêtements sauvagement ramifiés de courbes algébriques, Invent. Math. 141 (2000), no. 1, 195-238. MR 2001f:14023

2. Niels Borne, Cohomology of $G$-sheaves in positive characteristic, Adv. Math. 201 (2006), no. 2, 454-515. MR MR2211535

3. Helmut Boseck, Zur Theorie der Weierstrasspunkte, Math. Nachr. 19 (1958), 29-63. MR MR0106221 (21 \#4955) 
4. Wieb Bosma, John Cannon, and Catherine Playoust.The Magma algebra system. I. The user language. J. Symbolic Comput., 24 (3-4):235-265, 1997

5. David Eisenbud, Commutative algebra, Springer-Verlag, New York, 1995, With a view toward algebraic geometry.

6. Hershel M. Farkas and Irwin Kra, Riemann surfaces, Graduate Texts in Mathematics, vol. 71, SpringerVerlag, New York, 1980. MR MR583745 (82c:30067)

7. Arnaldo García, On Weierstrass points on Artin-Schreier extensions of $k(x)$, Math. Nachr. 144 (1989), 233239. MR MR1037171 (91f:14021)

8. $\ldots$, On Weierstrass points on certain elementary abelian extensions of $k(x)$, Comm. Algebra 17 (1989), no. 12, 3025-3032. MR MR1030607 (90m:14020)

9. Adolf Hurwitz, Über algebraische Gebilde mit eindeutigen Tranformationen in sich, Math. Ann. 41 (1893), 403-442.

10. N. Katz, Local to global extensions of representations of fundamental groups, Ann. Inst. Fourier (Gernoble) 36 (1986), no 4 69-106.

11. A. Kontogeorgis, Polydifferentials and the deformation functor of curves with automorphisms, Journal of Pure and Applied Algebra 210 (2007), no. 2, 551-558.

12. Aristides Kontogeorgis, Quadratic differentials and the deformation functor of curves with automorphisms, arXiv:math.AG/0610982

13. On the tangent space of the deformation functor of curves with automorphisms, Algebra Number Theory 1 (2007), no. 2, 119-161. MR MR2361938

14. Pedro Ricardo López-Bautista and Gabriel Daniel Villa-Salvador, On the Galois module structure of semisimple holomorphic differentials, Israel J. Math. 116 (2000), 345-365. MR MR1759412 (2001f:12007)

15. Lara Thomas, A valuation criterion for normal basis generators in equal positive characteristic, J. Algebra 320 (2008), no. 10, 3811-3820. MR MR2457723

16. Daniel J. Madden, Arithmetic in generalized Artin-Schreier extensions of $k(x)$, J. Number Theory 10 (1978), no. 3, 303-323. MR MR506641 (80d:12009)

17. Martha Rzedowski-Calderón, Gabriel Villa-Salvador, and Manohar L. Madan, Galois module structure of holomorphic differentials in characteristic p, Arch. Math. (Basel) 66 (1996), no. 2, 150-156. MR MR1367157 (97e:11142)

18. Joseph H. Silverman, Some arithmetic properties of Weierstrass points: hyperelliptic curves, Bol. Soc. Brasil. Mat. (N.S.) 21 (1990), no. 1, 11-50. MR MR1139554 (92k:11066)

19. Henning Stichtenoth, Algebraic function fields and codes, Springer-Verlag, Berlin, 1993. MR 94k:14016

20. Tuneo Tamagawa, On unramified extensions of algebraic function fields, Proc. Japan Acad. 27 (1951), 548551. MR MR0047705 (13,918a)

21. Christopher Towse, Weierstrass points on cyclic covers of the projective line, Trans. Amer. Math. Soc. 348 (1996), no. 8, 3355-3378. MR MR1357406 (96k:14024)

22. Robert C. Valentini, Representations of automorphisms on differentials of function fields of characteristic p, J. Reine Angew. Math. 335 (1982), 164-179. MR MR667465 (84j:12013)

23. Robert C. Valentini and Manohar L. Madan, Automorphisms and holomorphic differentials in characteristic $p$, J. Number Theory 13 (1981), no. 1, 106-115. MR MR602451 (83d:14011)

24. Steven H. Weintraub, Representation theory of finite groups: algebra and arithmetic, Graduate Studies in Mathematics, vol. 59, American Mathematical Society, Providence, RI, 2003. MR MR1984740 (2004k:20023)

Department of Mathematics, University of the Ægean, 83200 Karlovassi, Samos, Greece E-mail address: mathm03005@aegean.gr 